ARTICLE

\title{
Hedgehog-Interacting Protein is a multimodal antagonist of Hedgehog signalling
}

Samuel C. Griffiths (1) 1,5,6, Rebekka A. Schwab ${ }^{1,6}$, Kamel El Omari (10 2, Benjamin Bishop ${ }^{1}$, Ellen J. Iverson ${ }^{3}$, Tomas Malinauskas (10 ${ }^{1}$, Ramin Dubey ${ }^{3}$, Mingxing Qian ${ }^{4}$, Douglas F. Covey ${ }^{4}$, Robert J. C. Gilbert (1) ${ }^{1}$, Rajat Rohatgi (iD ${ }^{3} \&$ Christian Siebold (i) ${ }^{1 凶}$

Hedgehog $(\mathrm{HH})$ morphogen signalling, crucial for cell growth and tissue patterning in animals, is initiated by the binding of dually lipidated $\mathrm{HH}$ ligands to cell surface receptors. Hedgehog-Interacting Protein (HHIP), the only reported secreted inhibitor of Sonic Hedgehog (SHH) signalling, binds directly to $\mathrm{SHH}$ with high nanomolar affinity, sequestering $\mathrm{SHH}$. Here, we report the structure of the HHIP N-terminal domain (HHIP-N) in complex with a glycosaminoglycan (GAG). HHIP-N displays a unique bipartite fold with a GAG-binding domain alongside a Cysteine Rich Domain (CRD). We show that HHIP-N is required to convey full HHIP inhibitory function, likely by interacting with the cholesterol moiety covalently linked to $\mathrm{HH}$ ligands, thereby preventing this $\mathrm{SHH}$-attached cholesterol from binding to the $\mathrm{HH}$ receptor Patched (PTCH1). We also present the structure of the HHIP C-terminal domain in complex with the GAG heparin. Heparin can bind to both HHIP-N and HHIP-C, thereby inducing clustering at the cell surface and generating a high-avidity platform for $\mathrm{SHH}$ sequestration and inhibition. Our data suggest a multimodal mechanism, in which HHIP can bind two specific sites on the SHH morphogen, alongside multiple GAG interactions, to inhibit SHH signalling.

\footnotetext{
${ }^{1}$ Division of Structural Biology, Wellcome Centre for Human Genetics, University of Oxford, Oxford, UK. ${ }^{2}$ Science Division, Diamond Light Source, Harwel Science and Innovation Campus, Didcot, UK. ${ }^{3}$ Departments of Biochemistry and Medicine, Stanford University School of Medicine, Stanford, CA, USA.

${ }^{4}$ Department of Developmental Biology, Washington University School of Medicine, St. Louis, MI, USA. ${ }^{5}$ Present address: Evotec (UK) Ltd., Milton Park, Abingdon, UK. ${ }^{6}$ These authors contributed equally: Samuel C. Griffiths, Rebekka A. Schwab. ${ }^{凶}$ email: christian@strubi.ox.ac.uk
} 
T he Hedgehog $(\mathrm{HH})$ morphogen pathway fulfils crucial functions in growth and morphogenesis, whilst dysregulation leads to developmental disorders and cancer ${ }^{1-4}$. The secreted $\mathrm{N}$-terminal domain of $\mathrm{SHH}(\mathrm{ShhN})$ is generated from a $45 \mathrm{kDa}$ precursor, undergoing intein-based cleavage to couple an esterified cholesterol molecule to the $\mathrm{C}$-terminus ${ }^{5}$. A subsequent step involves the N-terminal attachment of a palmitoyl moiety to produce the fully active lipid-modified signalling ligand (palmitoylated and cholesteroylated $\mathrm{ShhN} ; \mathrm{pShhNc})^{6}$. Signalling is activated by binding of pShhNc to the extracellular domains of transmembrane protein PTCH1. Recent structural studies show that PTCH1 and pShhNc form a 2:1 complex, with one molecule of PTCH1 engaging pShhNc at a conserved high-affinity interface involving the conserved SHH zinc- and calcium-binding sites ("protein-protein interface"), and the other at the terminal SHHpalmitoyl and -cholesteryl moieties ("lipid interface") $)^{7-12}$. The PTCH1:pShhNc interaction releases inhibition of the G-protein coupled receptor Smoothened (SMO), which ultimately results in activation of target genes via the GLI transcription factors ${ }^{2}$. When no HH ligand is present, PTCH1 constitutively inhibits SMO signalling, potentially by preventing access to cholesterol or a similar sterol molecule ${ }^{10,13-16}$.

Extracellular distribution of $\mathrm{pShhNc}$ is key to the activation of correct signalling responses. This is controlled by a combination of co-receptor signalling ${ }^{17}$, the glycosaminoglycan (GAG) chains of heparan sulphate proteoglycans (HSPGs) ${ }^{18,19}$ and the assembly of pShhNc into multimers ${ }^{20,21}$. The HH pathway is modular, with several other essential cell surface receptors alongside PTCH1. For example, the immunoglobulin superfamily members CDO and $\mathrm{BOC}$ bind directly to $\mathrm{SHH}$ via the conserved interface involving the pShhNc metal-binding sites ${ }^{22-25}$. The metalbinding sites are also crucial for SHH interactions with the vertebrate-specific $\mathrm{HH}$ antagonist Hedgehog-Interacting Protein (HHIP) 26,27 . HHIP is the only secreted inhibitor of $\mathrm{HH}$ signalling 28-30, essential for the development of the lung ${ }^{31}$, cartilage $^{32}$ and brain $^{33}$. HHIP downregulation is associated with $\mathrm{HH}$-dependent tumourigenesis ${ }^{34}$ and variants at the HHIP locus are linked to Chronic Obstructive Pulmonary Disease (COPD), one of the most common devastating lung diseases in humans ${ }^{35}$. HHIP is composed of an N-terminal domain (HHIP-N) that shows weak sequence homology to the cysteine-rich domain (CRD) superfamily ${ }^{36}$, typically involved in small moleculebinding (Fig. 1a). The C-terminal domain of HHIP is composed of a $\beta$-propeller and two EGF repeats (HHIP-C). We and others previously determined structures of HHIP-C in complex with human $\mathrm{HH}$ ligands ShhN and DhhN, respectively ${ }^{26,27}$. HHIP utilises a loop inserted into blade 3 of its $\beta$-propeller to bind to the $\mathrm{HH}$ metal-binding site by directly coordinating the $\mathrm{Zn}$ ion. This suggests that HHIP inhibits HH function by sequestering the $\mathrm{HH}$ morphogen, and acts as a decoy receptor. Our recent study ${ }^{28}$ identified a role for HHIP-high affinity interaction with the GAG chains of heparan sulphate proteoglycans (HSPGs), as well as uncovering a cluster of residues in HHIP-N involved in this process. However, the role of the HHIP-N CRD and its potential small molecule binding properties remained elusive.

In this work, we use a combination of structural, biophysical and cellular studies to characterise the HHIP N-terminal region, revealing an unexpected GAG binding domain and a CRD with a small molecule-binding fold. We show that HHIP-N is necessary to convey they full signal inhibition by HHIP in response to pShhNc, and that the purified CRD binds to a mimic of the cholesteroylated HH C-terminus. Importantly, we also identify and structurally characterise two distinct GAG binding sites within HHIP-N and HHIP-C, respectively, and show that HHIPC assembles into large HHIP-GAG oligomers. Our results reveal that HHIP uses a modular mechanism for SHH inhibition. HHIP targets both the SHH metal-binding and lipid-modification sites recognised by PTCH1, while potentially staying localized on the cell surface via HHIP-GAG interactions.

\section{Results}

Structure of the HHIP N-terminal domain reveals a CRD fold. We expressed the N-terminal domain of HHIP (HHIP-N) using mammalian expression in HEK293T cells ${ }^{37}$ (Fig. 1a). Purified HHIP-N was crystallised in the presence of the GAG mimic sucrose octasulphate (SOS). The structure of the HHIP-N:SOS complex was determined using the single anomalous dispersion (SAD) method from native sulphur atoms. A multi-crystal approach was taken in which 24 data sets were collected from 8 isomorphous crystals, utilising both mini-kappa and inverse beam strategies to maximise the observed anomalous signal (Supplementary Table 1, Supplementary Fig. 1a-d) ${ }^{38,39}$. This allowed us to determine the structure of HHIP-N in complex with SOS at a resolution of $2.7 \AA$, with one molecule in the crystallographic asymmetric unit. HHIP-N possesses an elongated globular fold with a unique $\mathrm{N}$-terminal GAG-binding domain (GBD) and a C-terminal CRD (Fig. 1b). HHIP-N is stabilised by a total of 6 disulphide bonds. The GBD of HHIP-N binds SOS, and contains a single helix and flanking loop regions (Fig. 1b, lower panel) that are stabilised by one intra-domain disulphide bond (I: C39-78) and two inter-domain disulphide bonds with helix $\alpha 1$ of the CRD (II: C69-C112 and III: C79-115). The HHIP-N CRD is composed of 5 helices ( $\alpha 1-\alpha 5)$ stabilised by three intra-CRD disulphide bonds (IV: C103-C152, V: C141-179 and VI: C145-168). All disulphide bonds were identified as individual sites in the anomalous difference map, and treatment of these as 'super-sulphurs' was vital for substructure determination and structure solution (Fig. 1c) ${ }^{40}$.

CRDs can bind to various small molecules, including folate (folate receptors; $\mathrm{FR} \alpha / \beta)^{41}$ and riboflavin (riboflavin-binding protein; RFBP ${ }^{42}$, as well as lipids and sterols, such as cholesterol $(\mathrm{SMO}, \mathrm{NPC1})^{43,44}$ and palmitoleic acid (Frizzled; Fz) ${ }^{45}$. Previously, we performed an evolutionary structural analysis to classify known CRDs into two distinct sub-families, containing 'pockets' or 'grooves' as ligand-binding elements ${ }^{46}$. In this context, a 'pocket' refers to a CRD in which the ligand-binding site consists of loops that fold over a deep cavity inaccessible to solvent, whereas a 'groove' comprises a shallow and elongated solvent-accessible binding cleft, which does not undergo major conformational changes upon ligand binding. We have now included our HHIP-N structure in this analysis and classified it as a member of the pocket-type sub-family, with related structural homologues in the CRDs of NPC1, RFBP and FR $/ \beta$ (Fig. 1d, Supplementary Table 2). Additionally, this analysis identifies sperm-egg fusion protein JUNO as being a pocket-type CRD which has diverged from typical folate receptors ${ }^{47}$. The HHIP-N CRD comprises a structural 'scaffold' composed of helices a1, a3 and a5 that are stabilised by disulphide bridges, a feature common to all CRD family members (Fig. 1b, Supplementary Fig. 2). In our HHIP-N structure, we observed two stretches of disordered residues, which link the N-terminal domain and CRD helix al (DL1), and the loop regions between helices $\alpha 2$ and $\alpha 3$ (DL2), respectively (Fig. 2a). In FR $\beta$, the equivalent regions form the majority of a folate-binding pocket (Fig. $2 \mathrm{~b}$ ), suggesting that DL1 and DL2 very likely occupy a similar region. The modes of ligand binding by pocket-type CRDs are illustrated in Supplementary Fig. 3b, c and Supplementary Fig. 3f, while ligand binding by groove-type CRDs is detailed in Supplementary Fig. 3d-e. Helices analogous to HHIP-N a1-5 form an equivalent 'scaffold' in the pocket-type CRDs NPC1, RFBP and JUNO, within which 

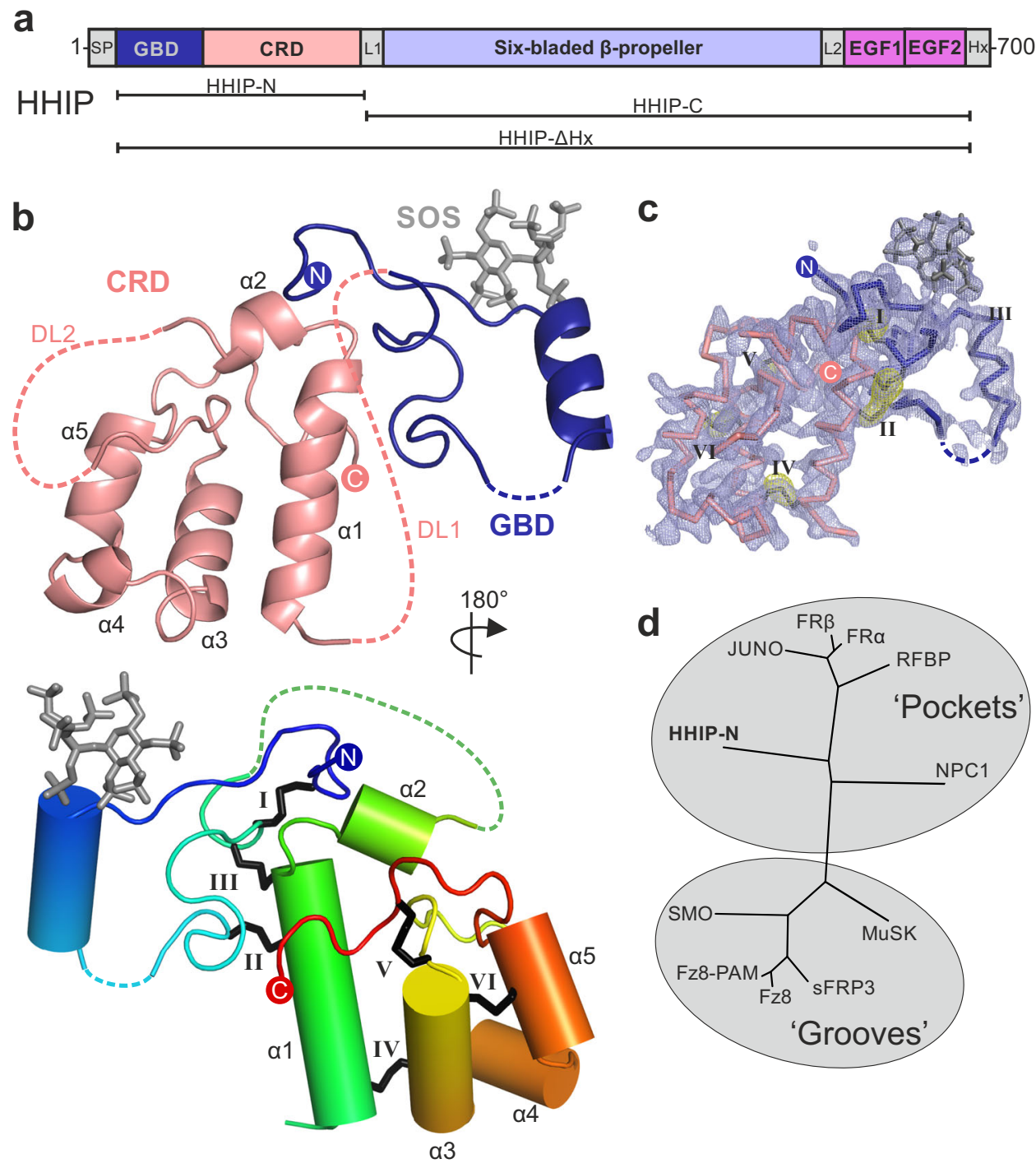

Fig. 1 The structure of HHIP-N. a HHIP domain organisation and construct design. b Cartoon representation of HHIP-N in complex with SOS. In the top panel, the CRD region is depicted in salmon and the N-terminal GAG-binding domain in dark blue. Two disordered loops (DL1 and DL2) are displayed as dashed salmon lines. In the lower panel, the HHIP-N:SOS complex is shown in rainbow colouring (blue: N-terminus, red: C-terminus). The CRD helices are labelled and disulphide bonds are numbered using Roman numerals. c Maps calculated from final HHIP-N model. The 2Fo-Fc map is shown in blue, contoured at $1 \sigma$. An anomalous difference map calculated from S-SAD data used for phasing is displayed as a yellow mesh contoured at $4 \sigma$. Corresponding disulphide bonds are numbered using Roman numerals. d Structural phylogenetic analysis of CRDs (see Supplementary Table 2).

ligand-binding loops are positioned for small molecule binding. A ligand-binding loop comparable to HHIP-N DL1 is also present in the groove-type CRDs of Fz8 and SMO (Supplementary Fig. 3d, e). This structural and evolutionary analysis suggests that loops DL1 and DL2 could potentially form a binding pocket for a physiologically-relevant small molecule ligand within the HHIP$\mathrm{N}$ CRD region.

Next, we tested the function of HHIP-N in a SHH signalling assay, based on measuring the mRNA levels of the $\mathrm{HH}$ target gene Gli1 in NIH/3T3 cells. We co-cultured PANC-1 human pancreatic ductal adenocarcinoma cells expressing $\mathrm{pShhNc}^{48}$ to activate the $\mathrm{HH}$ pathway. The purified full-length HHIP extracellular domain only lacking the C-terminal helix (residues 39-670, HHIP- $\Delta \mathrm{Hx}$, Fig. 1a) was able to inhibit $\mathrm{HH}$ signalling more efficiently than HHIP-C (Fig. 2c), which lacks HHIP-N but contains the high-affinity SHH binding site ${ }^{26,27}$. We next asked whether the interaction of HHIP-N with the SHH lipid modifications could account for this increase of inhibition, given the structural similarities with small molecule-binding
CRDs. For this, we activated the $\mathrm{HH}$ pathway with an N-terminal palmitoylated-SHH peptide (Palm-ShhN15) ${ }^{49}$, which does not interact with HHIP-C. In this context, HHIP$\Delta \mathrm{Hx}$ was unable to inhibit signalling (Fig. $2 \mathrm{~d}$ ), excluding a role of the SHH N-terminal palmitoyl moiety in HHIP-N binding. We recently showed that the $\mathrm{SHH}$ C-terminal cholesterol moiety is important for SHH-PTCH1 interactions and activation of $\mathrm{HH}$ signalling ${ }^{12}$. Using a similar isothermal titration calorimetry (ITC) assay, we tested whether a PEGylated cholesterol molecule (mimicking the C-terminus of pShhNc) binds to HHIP. PEG-cholesterol bound specifically to HHIP-N $\left(K_{D}=23 \mu \mathrm{M}\right)$ (Fig. 2e), whereas free PEG did not bind to HHIP-N (Fig. 2f). Due to the high heat of dilution when PEGcholesterol is titrated into buffer, a series of controls were conducted (Supplementary Fig. 4). Thermodynamic signature plots for HHIP-N compare favourably with those obtained for the recently published interaction of PEG-cholesterol with the canonical $\mathrm{Hh}$ receptor $\mathrm{PTCH} 1^{12}$, revealing a high enthalpic contribution to $\Delta \mathrm{G}$ suggesting a hydrophobic interaction ${ }^{50}$. 

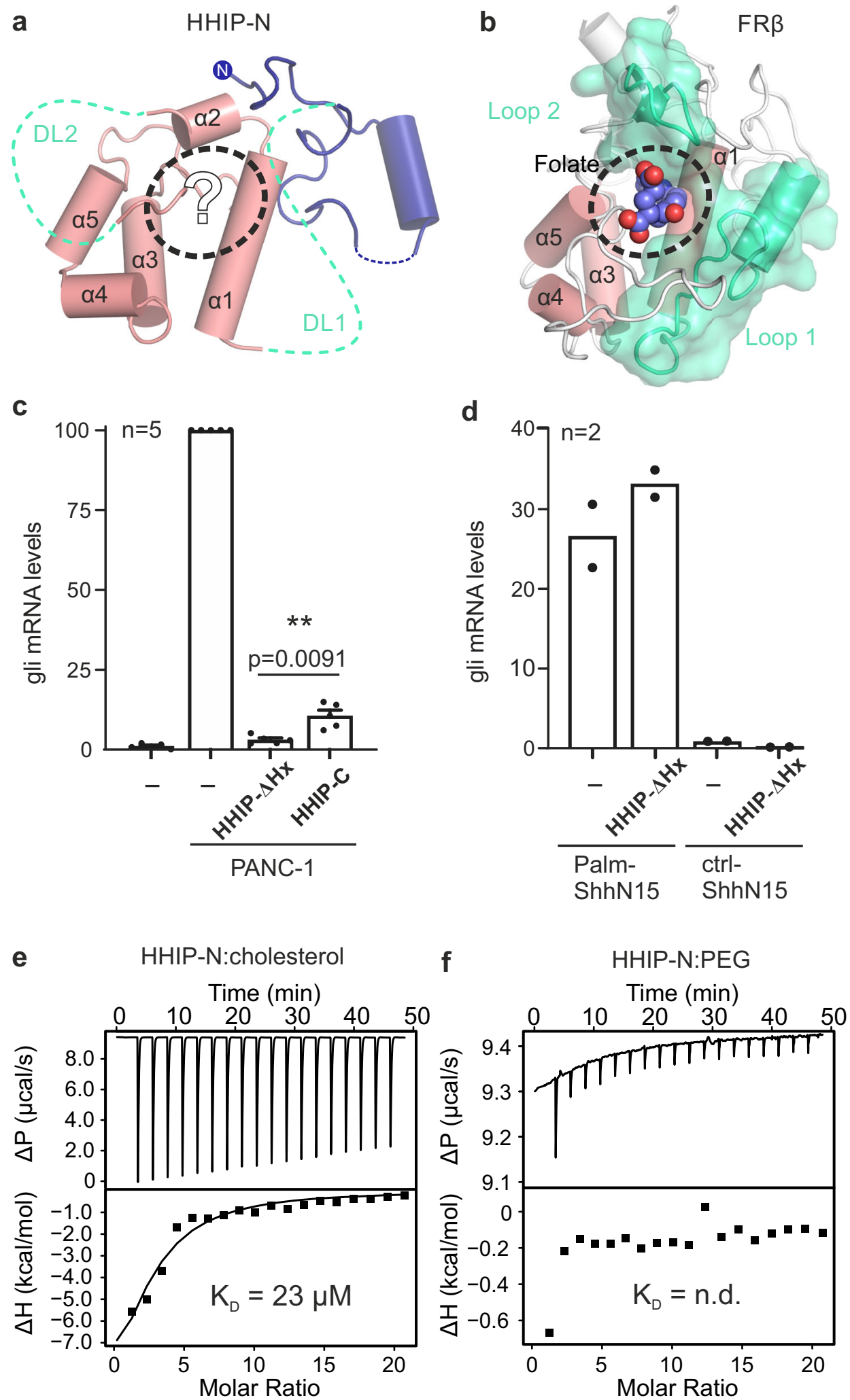

This supports that the interaction between PEG-cholesterol and HHIPN is specific. Taken together, the enhancement of $\mathrm{HH}$ signalling inhibition by HHIP in the presence of HHIP-N, combined with structural homology to small molecule-binding pocket-type CRDs and observed binding to PEG-cholesterol suggests an interaction between HHIP-N and $\mathrm{HH}$-linked cholesterol. Hence, we propose a model whereby HHIP binds

to $\mathrm{SHH}$ in a multimodal manner, utilising the $\mathrm{SHH}$ metalbinding sites for HHIP-C binding and potentially targeting the cholesterol attachment site for HHIP-N binding. Such an arrangement could be accommodated in a 1:1 SHH-HHIP complex, as HHIP-N and HHIP-C are separated by a $25-$ residue long, likely flexible linker that could position the SHHlinked cholesterol to interact with HHIP-N and the SHH-metal 
Fig. 2 HHIP-N CRD contributes to HH signalling inhibition. a, b Structural homology of HHIP-N with CRDs suggests small molecule interaction. HHIP-N (a) is shown, with missing loops DL1 and DL2 displayed as green dashed lines and helices are annotated $\alpha 1-5$. A potential ligand-binding pocket is outlined with a dashed circle. FR $\beta$ (b) binds a folate molecule (spheres, circled), utilising loops structurally analogous to HHIP-N DL1 and DL2 (coloured green). Helices are annotated $\alpha 1-5$ as in HHIP-N. c RT-PCR assay from NIH/3T3 cells co-cultured with PANC-1 cells expressing pShhNc to quantify Hh signalling in the presence of HHIP constructs. Relative levels of Gli1 mRNA were quantified and normalised from 5 independent experiments and displayed as mean values $\pm \mathrm{SEM}$, with statistical significance calculated using a two-tailed, paired t-test with $p=0.0091$. $\mathbf{d} \mathrm{HH}$ signalling assay to assess $\mathrm{HHIP}$ - $\Delta \mathrm{Hx}$ inhibition of pathway activation in response to a palmitoylated N-terminal pShhNc peptide. e Raw ITC (upper panel) and binding isotherm (lower panel) for titration of PEG-cholesterol into HHIP-N. f Raw ITC (upper panel) and binding isotherm (lower panel) for titration of unconjugated PEG200 into HHIP-N. Source data are available as Source Data file.

binding site to interact with HHIP-C within the same molecule (Supplementary Fig. 5).

The HHIP N-terminal domain contains a GAG-binding domain. To shed light onto the previously reported GAGbinding properties of HHIP- $\mathrm{N}^{28}$, we determined structures of apo and SOS-bound HHIP-N to resolution of $2.6 \AA$ and $2.7 \AA$, respectively (Supplementary Table 1). In the apo HHIP-N structure, the N-terminal GAG-binding domain is disordered, while the CRD region is inherently structured (Fig. 3a). This suggests that interaction of HHIP-N with GAG molecules triggers a transition to a folded state, forming an $\alpha$-helix between residues 50 and 58. Each HHIP-N molecule interacts with 3 SOS molecules (Fig. 3b, Supplementary Fig. 6). SOS is bound to a positively charged surface localised to the HHIP-N N-terminal domain (Fig. 3c), forming electrostatic interactions with a cluster of 6 basic residues and burying a total surface area of $594 \AA^{2}$ (Fig. 3d). Only Arg-47 forms hydrogen bonds with more than one SOS molecule; Arg-51 and Arg-54 are the only residues within the $\mathrm{N}$-terminal $\alpha$-helical residues $50-58$ to contact a SOS molecule. Mutagenesis of SOS-interacting basic HHIP residues (marked with asterisks in Fig. 3d) were previously observed to weaken the interaction between HHIP and heparin, validating our observed HHIP-N:SOS interface ${ }^{28}$. The N-terminal domain of HHIP-N is different compared to other structurally characterised CRDs, having evolved a GAG-binding function in a discrete domain alongside a conserved small molecule-binding fold. Additionally, we report structural insights into the major secondary structural rearrangements of HHIP-N, a GAG-binding protein, upon sugar coordination.

Structure of the HHIP C-terminal domain in complex with heparin. Our previous analysis of HHIP:GAG interactions identified multiple GAG-binding motifs in HHIP, since a construct that contains a deletion of HHIP-N was still able to bind to heparin ${ }^{27}$. To decipher the GAG-binding properties of HHIP, we conducted binding assays using three domain deletion constructs (Fig. 1a). First, we tested GAG binding using heparin affinity chromatography (Fig. 3e). Both HHIP-C and HHIP-N show binding to a heparin column, albeit with weaker affinity when compared to HHIP- $\Delta$ Hx. Furthermore, HHIP-N, HHIP-C and HHIP- $\Delta \mathrm{Hx}$ are all cell surface-associated at physiological $\mathrm{pH}$ and ionic strength (Supplementary Fig. 7). Taken together, our analysis suggests that both HHIP-N and HHIP-C contribute to the observed affinity for GAG chains.

To structurally characterise HHIP-C:GAG interactions, we determined the $2.7 \AA$ resolution crystal structure of HHIP-C in complex with a 30-mer heparin molecule (Supplementary Table 1, Supplementary Fig. 8a). HHIP-C comprises an N-terminal 6bladed $\beta$-propeller and $2 \mathrm{C}$-terminal EGF repeats, previously identified in the complex of HHIP-C with HhN ligands 26,27 . The heparin chain contacts two separate clusters of HHIP surface residues (GAG 'sitel' and GAG 'site 2') at either end of the molecule (Fig. 4a, Supplementary Fig. 8). In this arrangement, a HHIP-C anti-parallel dimer coordinates one single heparin chain (Fig. 4b) by using a central positively-charged region formed by residues from both HHIP protomers (Fig. 4c). The HHIP-C chains in the dimer are essentially identical (r.m.s.d. of $0.21 \AA$ for 322 equivalent $\mathrm{C} \alpha$ atoms), and show little structural difference to previously published HHIP-C structures (e.g. r.m.s.d. of $0.31 \AA$ for 331 equivalent $\mathrm{C} a$ atoms, PDB ID. 2WFT ${ }^{26}$ ). The heparin backbone displays a right-handed helical structure with roughly 4 sugars per turn, in agreement with previous structural studies $^{51,52}$. A total of 8 monosaccharides are resolved in the structure, running from a reducing (O1; sugar $\mathrm{I})$ to a nonreducing end (O4; sugar VIII) and forming hydrogen bonds with several polar side chains (Fig. 4d). We also determined the crystal structure of a HHIP-C:SOS complex, which exhibits the equivalent anti-parallel, dimeric HHIP arrangement bound to two SOS molecules coordinated at the same site compared to heparin (Supplementary Table 1, Supplementary Figs. 8b, 9 and 10). In summary, our HHIP-C crystal structures in complex with GAG molecules identify two discrete GAG-binding sites on the surface of HHIP-C, which are distinct and non-overlapping with the HhN-binding interface (Fig. 4e), thus suggesting that both GAG- and HhN-binding can occur simultaneously.

GAG interactions control oligomerisation of HHIP. Our previous study on HHIP:GAG interactions identified low micromolar affinities for the interactions between HHIP-C and the GAGs heparin, heparan sulphate (HS) and chondroitin sulphate $(C S)^{28}$. To experimentally validate our HHIP-C:GAG complex structures, we generated a HHIP mutant in which 6 positivelycharged residues involved in GAG recognition were mutated to glutamate (K277E/R328E/R350E/K569E/R610E/R613E, HHIP-C Glu mutant; Fig. 4d) and analysed binding to GAGs. Using surface plasmon resonance (SPR) with GAGs immobilised on the chip, we observed that the HHIP-C Glu mutant almost completely abolishes binding to heparin, HS and CS, when compared to wild-type (HHIP-C WT) (Fig. 5a). Furthermore, we analysed our observed HHIP-C:GAG interfaces by a combination of mutagenesis and heparin affinity chromatography. In addition to the HHIP-C Glu mutant, we generated glycosylation mutants (Supplementary Fig. 10a). N-linked glycans were inserted into the GAG-interacting surfaces of either the HHIP $\beta$-propeller (' $\triangle \mathrm{GAG}$ site 1') or EGF repeats (' $\triangle \mathrm{GAG}$ site $2^{\prime}$ ), as well as combination of both (' $\triangle$ GAG sites $1+2$ '). As expected, all mutants showed reduced heparin binding affinity relative to HHIP-C WT (Supplementary Fig. 11b). Next, we tested the effect of HHIP-C WT or HHIP-C Glu mutant in SHH signalling assays using NIH/3T3 cells (Fig. 5b). We observed that the HHIP-C Glu mutant showed significantly less efficient $\mathrm{SHH}$ inhibition. To further probe whether this effect results from loss of GAG-binding, we knocked out EXTL3, a key enzyme in the biosynthesis of cell-surface heparan sulphate (HS) chains (Supplementary Fig. 12a-d) ${ }^{53}$. We confirmed that single Extl3-1- NIH/3T3 clones lack surface HS chains by showing markedly reduced binding to a scFv antibody 
a

b
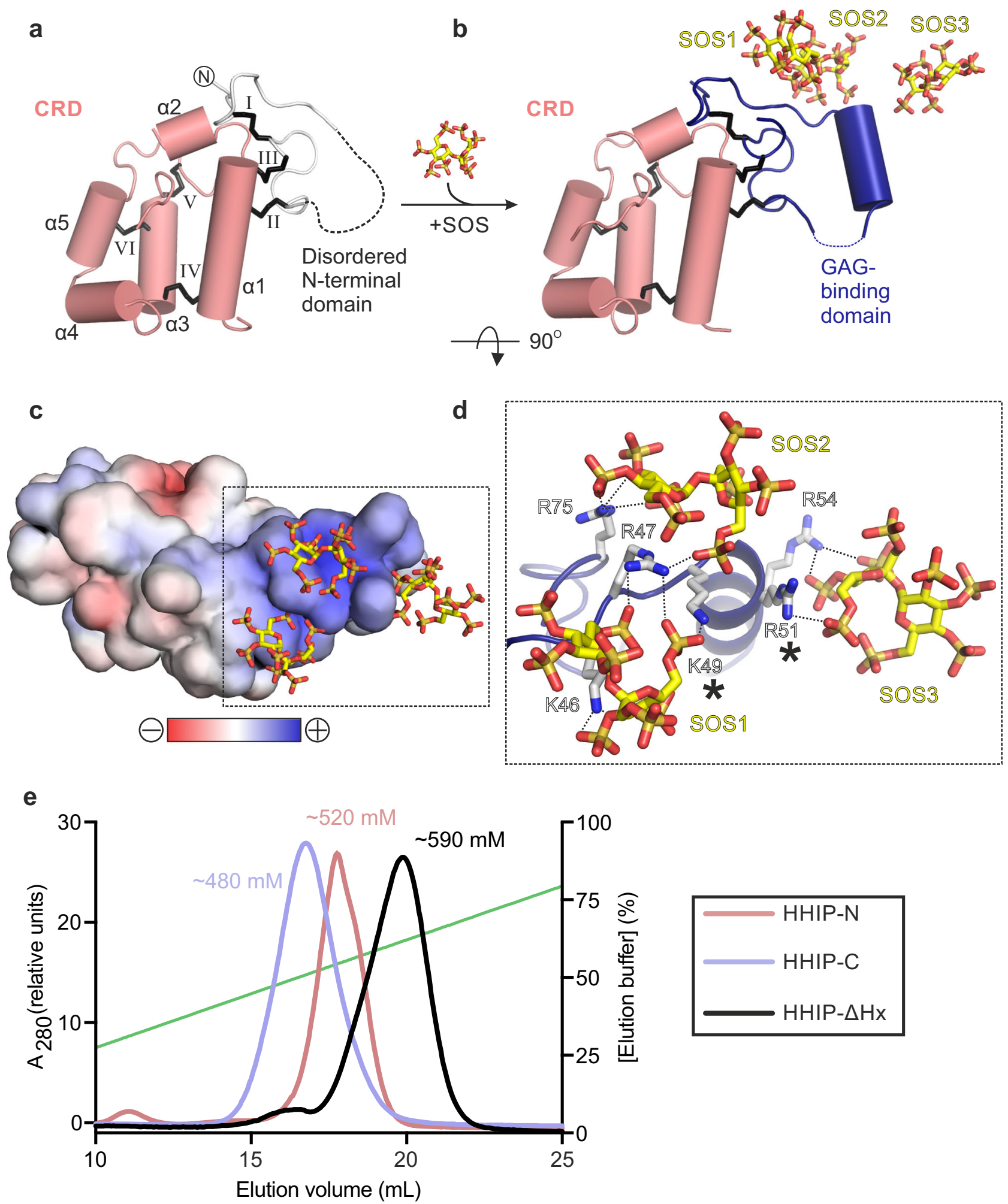

Fig. 3 HHIP-N:GAG interactions. a, b Comparison of the apo HHIP-N structure (a) and HHIP-N:SOS complex. Colour coding is as in Fig. 1b. For HHIP$\mathrm{N}$ :apo, the disordered GAG-binding domain is shown as a dashed line. In the HHIP-N:SOS complex three SOS molecules interact with the GAG-binding domain. c Electrostatic surface potential of the HHIP-N:SOS complex, displayed from $-10 \mathrm{kT} / \mathrm{e}$ to $+10 \mathrm{kT} / \mathrm{e}$. d Close-up view of HHIP-N:SOS binding site showing the region marked in $\mathrm{C}$. Hydrogen bonds are shown as dashed lines (c, $\mathbf{d}$ rotated $90^{\circ}$ around the $\mathrm{x}$-axis relative to $\mathrm{B}$ ). Mutations previously observed to decrease GAG-binding affinity and cause dysfunction of HHIP are marked with asterisks ${ }^{28}$. e Heparin affinity chromatography of different HHIP constructs eluted from a linear gradient of $\mathrm{NaCl}$ from 20 to $1000 \mathrm{mM}$ (green line). Traces corresponding to different constructs are coloured according to the accompanying legend (inset). 
a

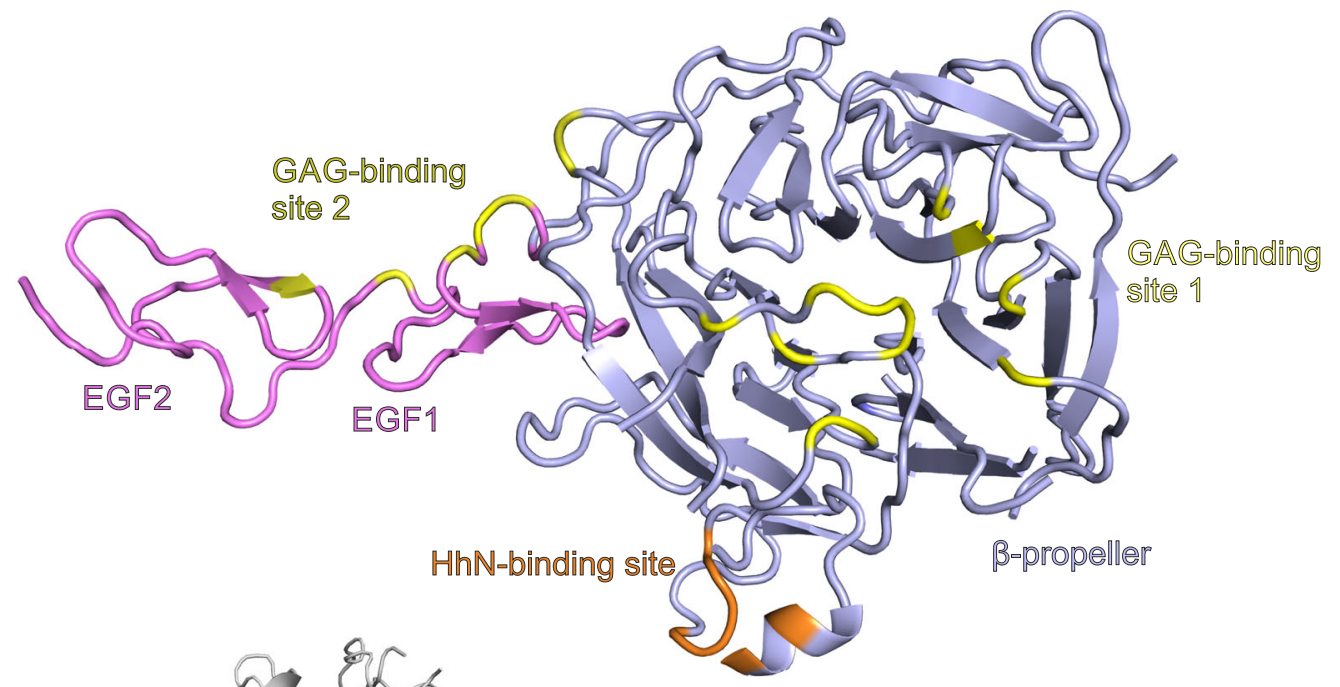

b
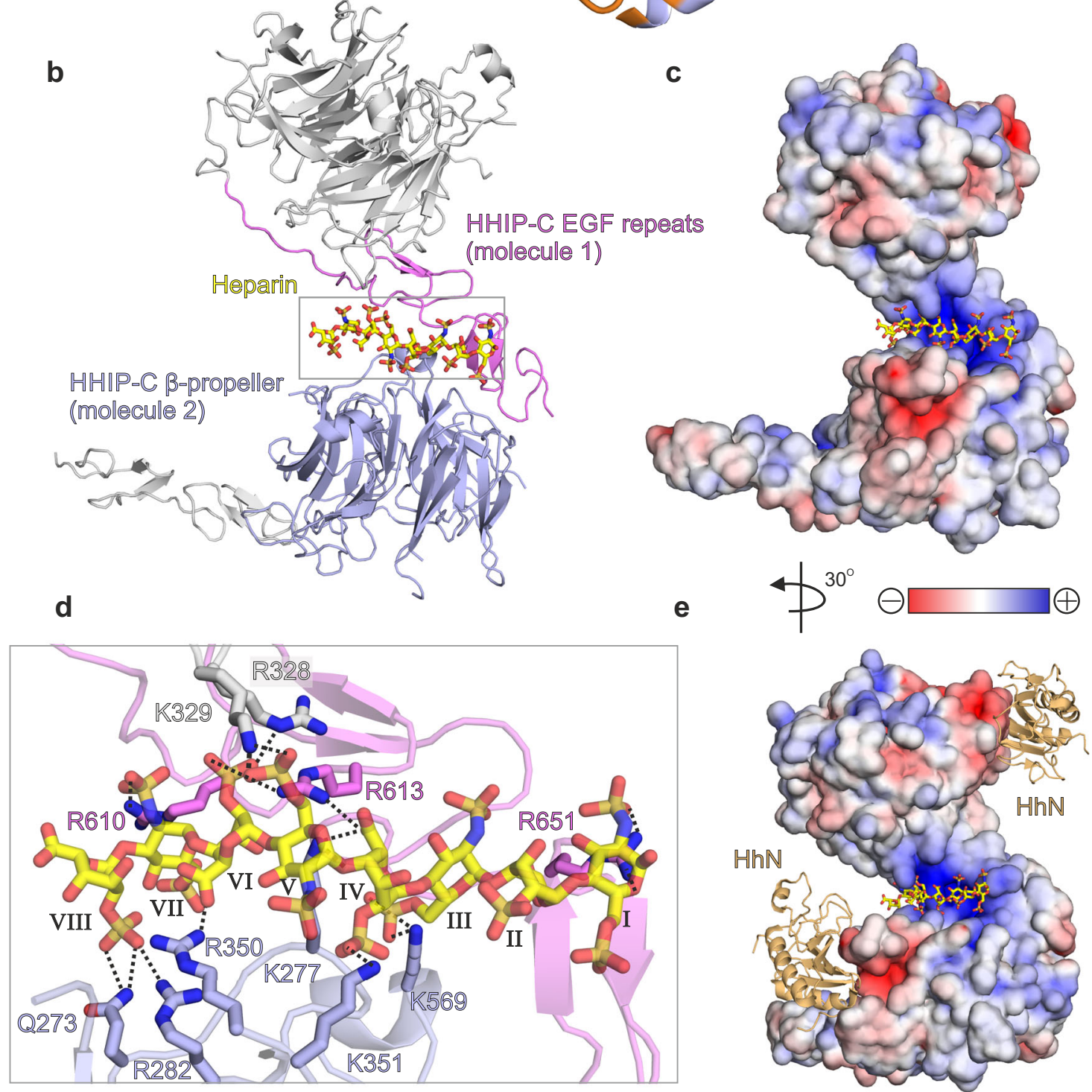

Fig. 4 The structure of the HHIP-C:heparin complex. a Cartoon representation of the HHIP-C monomer structure with the $\beta$-propeller domain in slate, the EGF repeats in magenta, the GAG-binding sites in yellow and the HhN-binding site in orange. $\mathbf{b}$ Arrangement of the two HHIP-C molecules (cartoon representation) around a centrally-coordinated heparin molecule (stick representation). c Close-up view of the boxed region in $\mathrm{B}$, displaying side chain:heparin interactions. Hydrogen bonds are displayed as black dashed lines and sugar residues are numbered using roman numerals (odd, N,O6disulphoglucosamine; even, O2-Sulphoiduronic acid). d Electrostatic surface potential shown from red ( $-8 \mathrm{kT} / \mathrm{e})$ to blue ( $+8 \mathrm{kT} / \mathrm{e})$ with heparin chain shown as sticks. e Comparison of heparin (yellow sticks) and HhN (orange cartoon) interaction sites on HHIP-C electrostatic surface. 
a

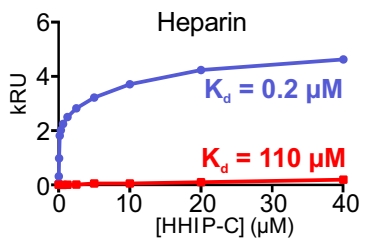

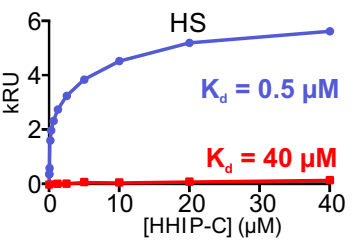

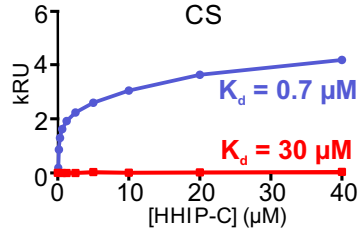

b

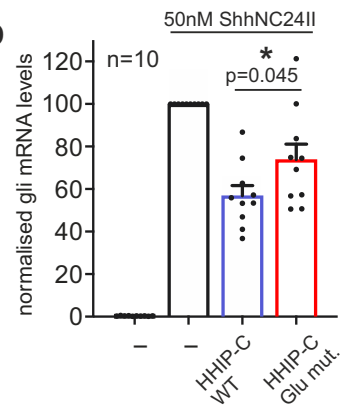

C

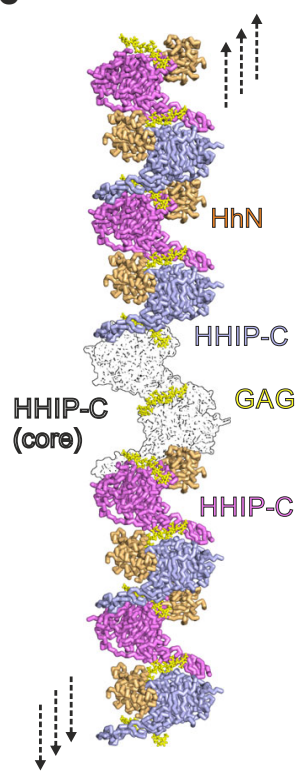

d

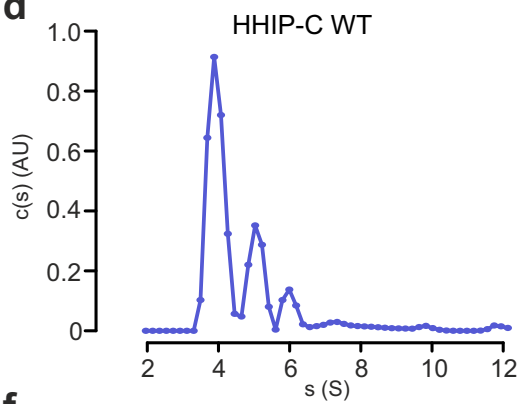

f

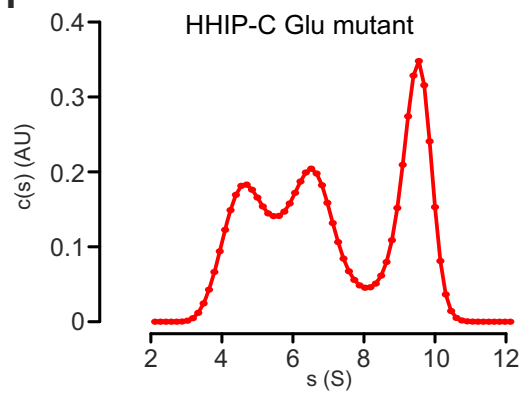

e

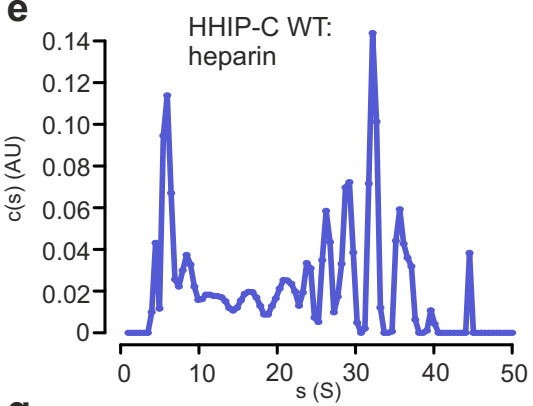

g

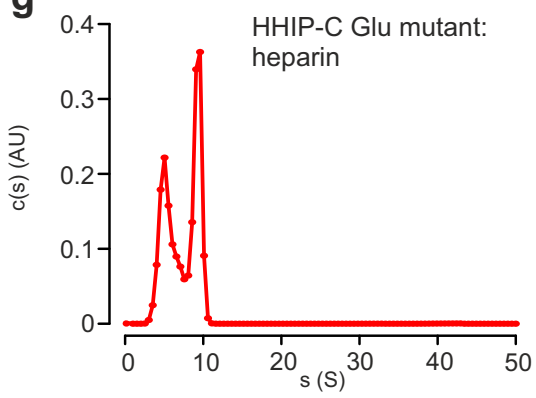

Fig. 5 Analysis of HHIP-C:GAG interactions and oligomerisation. a Comparative SPR curves for HHIP-C WT (light blue) and HHIP-C Glu mutant (red) binding to heparin, heparan sulphate (HS) and chondroitin sulphate (CS); b Quantification of HH pathway inhibition with the addition of HHIP-C WT or HHIP-C Glu mutant. Relative levels of Gli1 mRNA were quantified from 10 independent experiments, with statistical significance calculated using a twotailed, paired t-test with $p=0.045$; Data are presented as mean values \pm SEM. c Model for cell surface HHIP-C oligomeric assembly. GAG chains (represented by heparin, yellow) stabilise a core anti-parallel HHIP-C dimer (surface; black and white) and can then recruit further HHIP-C chains (light blue, violet) to form long oligomeric chains. This facilitates binding and antagonism of multiple HhN ligands (orange). d, e AUC experiments to assess the oligomerisation of HHIP in solution, comprising HHIP-C (d) and HHIP-C:heparin 30-mer complex (e); f, g As in (c, d), comprising HHIP-C Glu mutant (e) and HHIP-C Glu mutant mixed with 30-mer heparin (f). Source data are available as Source Data file.

(HS20) that is known to recognize multiple HS chains ${ }^{54,55}$ (Supplementary Fig. 12e). These Extl3-l- clones were responsive to $\mathrm{SHH}$, showing that the machinery required to receive and respond to ligands was unaffected by the loss of HS chains (Supplementary Fig. 12e). Compared to their individual level of $\mathrm{SHH}$ activation, wildtype HHIP was a less effective inhibitor in both knock-out cell lines than in their parental cell line. Even more importantly, both HHIP wildtype and Glu mutant inhibited the $\mathrm{HH}$ response in the HS-deficient cells to similar degrees, in contrast to the parental cell line where Glu mutant HHIP was a less effective inhibitor (Supplementary Fig. 12e). This demonstrates that the interaction between HHIP-C and GAGs are critical for maximal $\mathrm{HH}$ pathway inhibition.

Our HHIP-C:heparin complex structure also suggests that GAGs can mediate the formation of long oligomeric chains of HHIP (Fig. 5c). We observe two HHIP oligomerisation interfaces - an anti-parallel 'head-to-tail' heparin-bound HHIP-C dimer (Supplementary Fig. 13a) with a total buried surface area of $603 \AA^{2}$ (Supplementary Fig. 13a), and a 'head-to-head' dimer with a buried surface area of $981 \AA^{2}$ (Supplementary Fig. 10b) in the crystal. The 'head-to-tail' dimer interface is smaller than physiological dimer interfaces and is likely stabilised by the heparin molecule (PISA $\Delta^{\mathrm{i}} \mathrm{G}$
$P$-value $=0.41$, shape complementarity score $=0.75)^{45,46}$, while the larger 'head-to-head' interface is more likely to be physiological (PISA: $\Delta^{\mathrm{i} G} P$-value $=0.43$, shape complementarity score: 0.52 ). Previous studies using multi-angle light scattering (MALS) showed that the apo HHIP-C is monomeric at a concentration of approximately $10 \mu \mathrm{M}^{26}$. To test whether oligomers observed in the crystal structure exist in solution at higher concentrations (potentially mimicking the local concentrations at the cell membrane), we performed sedimentation velocity analytical ultracentrifugation experiments (AUC). This revealed a predominantly monomeric apo HHIP-C population $(4 \mathrm{~S}, 53 \mathrm{kDa})$, with minor populations of dimers $(5 \mathrm{~S}, \sim 100 \mathrm{kDa})$ and trimers $(6 \mathrm{~S}$, $\sim 150 \mathrm{kDa}$ ) also observed (Fig. 5d). As expected, addition of the heparin 30-mer triggers a drastic shift to increased sedimentation rates, and causing the sedimentation of an array of higher-order oligomeric species from $50 \mathrm{kDa}$ up to $6 \mathrm{MDa}$ (4 S-50 S; Fig. 5e). Higher-order oligomer formation in response to the addition of heparin is completely ablated in the HHIP-C Glu mutant (Fig. $5 \mathrm{f} \pm \mathrm{g}$ ), consistent with GAG-binding induced oligomerisation of HHIP. Taken together, our biophysical and cellular data suggest that HHIP:GAG complexation leads to HHIP clustering, and these assemblies might fine-tune inhibition of the $\mathrm{HH}$ signal. 
a Signalling complex Negative receptor complex

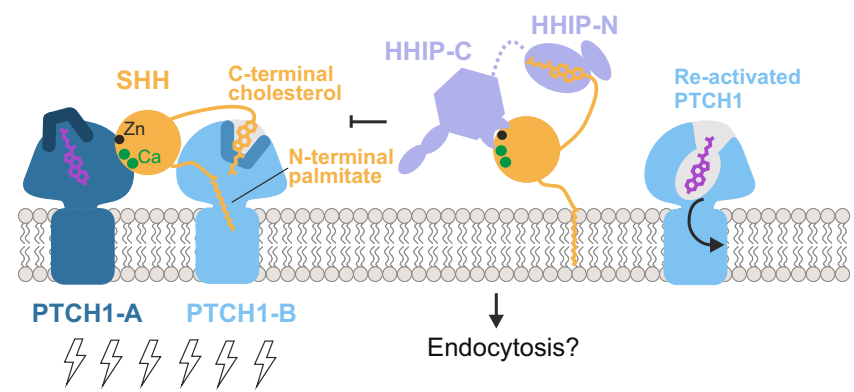

c HHIP:GAG oligomers

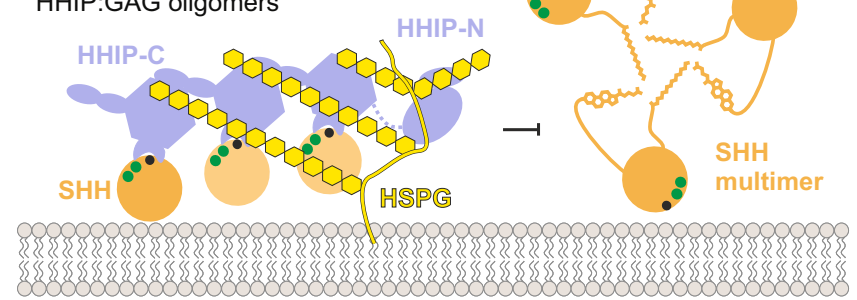

Fig. 6 Models of extracellular HH signalling antagonism by HHIP. a A 2:1 interaction of PTCH1:pShhNc represents the active signalling complex. The pShhNc lipid appendages insert to seal channels in PTCH1-B. b HHIP-C targets the conserved high-affinity metal ion-binding site on the pShhNc surface ('protein-protein interaction'), competing with binding from PTCH1A and other co-receptors, whilst HHIP-N is able to contribute to this by targeting the $\mathrm{C}$-terminal cholesterol moiety, possibly re-establishing $\mathrm{PTCH} 1$ cholesterol transport ability. It is also of note that $\mathrm{HH}$ signalling is activated via SMO CRD-cholesterol interaction, and competition of this by the HHIP$\mathrm{N}$ CRD represents a possible additional mode of inhibition. Additionally, $\mathrm{HHIP}$-mediated receptor endocytosis may act to remove $\mathrm{SHH}$ from the cell surface. c Cell surface GAG-mediated clustering represents a second modality for $\mathrm{HH}$ regulation by HHIP. The avidity of HHIP is increased by cluster formation at the proteoglycan layer, similar to how $\mathrm{SHH}$ lipoprotein multimers act to increase local morphogen concentration. The GAGbinding site in HHIP-N is also important for this process.

HHIP-N can also bind GAGs with high affinity and despite crystallising as a monomer, forms weak dimers in solution when analysed using MALS (Supplementary Fig. 14a). Interestingly, apo HHIP-N is predominantly monomeric in solution up to $200 \mu \mathrm{M}$ when studied by AUC (Supplementary Fig. 14b), and can also form oligomers in the presence of heparin (up to a size consistent with tetramers, as shown from $\mathrm{c}(\mathrm{s}, \mathrm{f} / \mathrm{f} 0)$ plots to analyse molecular weights in solution; Supplementary Fig. 14c). This is consistent with our crystal packing analysis (Supplementary Fig. 4), and suggests that GAGs stabilise a contact between HHIP$\mathrm{N}$ GAG-binding domains in solution to assist oligomerisation (Supplementary Fig. 14d). In conclusion, HHIP is regulated through both protein:protein- and protein:GAG-mediated oligomerisation at several different sites, that can be linked to dynamic modulation of $\mathrm{HH}$ signalling.

\section{Discussion}

The function of the N-terminal domain of HHIP has remained a long-standing mystery. Previous work in our laboratory and by others showed that HHIP-C binds to the metal-binding site of $\mathrm{SHH}$ with high (low nanomolar) affinity, and inhibits $\mathrm{HH}$ signalling by functioning as a decoy receptor ${ }^{26}$. Our structural, biophysical and cellular work reveals that HHIP-N contains a CRD, which acts as an additional module within HHIP to inhibit $\mathrm{HH}$ signalling. Moreover, HHIP-N has evolved a structured region engaged in cell surface heparan sulphate proteoglycan binding. Recent pioneering work on the PTCH1:pShhNc interaction has greatly enhanced our understanding of $\mathrm{HH}$ signal reception and transduction. $\mathrm{PTCH} 1$ and $\mathrm{SHH}$ interact in a 2:1 stoichiometry, with one PTCH1 molecule (PTCH1-A) binding to the high-affinity metal-binding site of $\mathrm{SHH}$ and the other PTCH1 molecule (PTCH1-B) grasping the two lipid modifications to form the full signalling complex (Fig. 6a). None of these interactions alone is sufficient to fully inactivate PTCH1 function in cellular assays ${ }^{12}$. This mechanism shows parallels to WNT morphogen signalling, in which WNT interacts with its receptor Frizzled (Fz) with both its covalently-linked palmitoleate (via the Fz-CRD, evolutionarily related to the HHIP-N CRD), and also via a protein-protein interface ${ }^{45}$. WNT inhibition is, in part, achieved by interactions with secreted Fz-related proteins (sFRPs) (via both palmitoleate- and protein-protein contacts), which act as secreted decoy receptors ${ }^{56}$. Our structural and functional analysis suggests that HHIP has potentially evolved a similar role, targeting the $\mathrm{SHH}$ cholesterol moiety (via HHIP-N) and the metalbinding site (via HHIP-C). Thus, a two-pronged engagement with both protein- and lipid-based interfaces seems to be a common theme in recognition of morphogens.

Insertion of the SHH palmitoyl and cholesteryl moieties into the ectodomain of PTCH1-B blocks a proposed conduit for cholesterol/sterol transport ${ }^{57,58}$. Shielding of the pShhNc cholesterol modification by HHIP-N would be an effective mechanism to release $\mathrm{SHH}$-mediated inhibition and transport function of PTCH1. This is complemented by HHIP-C and PTCH1-A competing for the SHH metal-binding site, which also overlaps with the binding sites for co-receptors CDO, BOC and GAS1 $1^{17,22}$. These two modes of signal antagonism constitute a fail-safe mechanism by which $\mathrm{HHIP}$ regulates $\mathrm{HH}$ signalling. HHIP-C binds to the SHH metal-containing site some 100-fold tighter compared to $\mathrm{CDO}^{22,26}$ and PTCH1 ${ }^{12}$, and thus could outcompete both CDO and PTCH1 from this site. This could result in the formation of a 'negative receptor complex,' facilitating for example $\mathrm{SHH}$ endocytosis (Fig. 6b).

Various studies have identified HHIP as the only known secreted diffusible inhibitor of $\mathrm{HH}$ signalling $28,29,59$. We previously showed that HHIP secretion is dependent on interaction with GAGs, and that HHIP can bind to various types of GAGs ${ }^{28}$. Here, we have delineated the molecular basis for this interaction. Both HHIP-N and HHIP-C contain discrete GAG-binding sites, which combine to modulate cell surface affinity. Together, they constitute additional minor regulatory sites of $\mathrm{HH}$ signalling. Cell surface-attached GAGs such as HS and CS organise secreted proteins into gradients, varying local concentrations to enable graded signal activation ${ }^{60}$. Here, we show that GAGs are able to cluster HHIP-C into large assemblies, linked to the potency of HHIP-mediated $\mathrm{HH}$ inhibition (which is further potentiated by HHIP-N). In addition, the HHIP C-terminal helix (residues 671-700) that contributes to cell surface binding via formation of a predicted leucine zipper-type assembly ${ }^{28}$ may enhance clustering even further, combined with the dimerisation abilities observed for both HHIP-N and HHIP-C. HHIP clustering by GAGs at the cell surface generates a high-avidity platform for $\mathrm{SHH}$ binding. This platform might limit diffusion and loss of HHIP into extracellular space and position HHIP to inhibit SHH signalling at the cell surface (Fig. $6 \mathrm{c}$ ). This process can be further regulated by $\mathrm{SHH}$-mediated internalisation of $\mathrm{HHIP}^{29}$, which potentially can happen at both $\mathrm{SHH}$ producing or responding cells. From our structural analysis, we also note that there is a long flexible linker between HHIP-N and HHIP-C, which we previously described as being proteolytically sensitive ${ }^{26}$. Cleavage of this linker may regulate the overall cell surface affinity of HHIP in vivo. Taken together, our results indicate that HHIP regulation 
of HH signalling is dynamic, and inhibitory processes are modularised through distinct functionalities within the HHIP N- and C-terminal domains.

Aberrant expression of SHH has been linked to the initiation and progression of numerous cancers, and $\mathrm{HH}$ inhibitors targeting SMO are in the clinic against basal cell carcinomas ${ }^{3,4}$. The SHH-specific mouse antibody 5E1 binds tightly to the SHH metal-binding site, overlapping with $\mathrm{HHIP}^{61}$, and has been shown to inhibit $\mathrm{SHH}$ activity in vivo and to reduce tumour growth in a pancreatic cancer mouse model ${ }^{62}$. HHIP could be used in a similar way, working as an engineered biologic to inhibit $\mathrm{HH}$ signalling. Here, we provide the framework for the development of HHIP-based HH inhibitors that can specifically preserve or block the different SHH-receptor interaction sites, and this interaction mode can be further fine-tuned by the GAGbinding properties and resultant HHIP clustering. Various biomedically-important secreted signalling proteins are reported to undergo GAG-dependent clustering 63 , and drugging such mechanisms could present an unexploited avenue for therapeutic discovery.

\section{Methods}

Protein expression and purification. Constructs of human HHIP (UniProt ID: Q96QV1) comprising the N-terminal domain (HHIP-N, 39-209), C-terminal domain (HHIP-C, 213-670) ${ }^{26}$ and a full-length variant only lacking the C-terminal helix (HHIP- $\Delta \mathrm{Hx}$; 39-670) were cloned in the pHLsec vector in frame with a $\mathrm{C}$-terminal $\mathrm{His}_{6}-\mathrm{tag}^{37}$. Additionally, HHIP- $\triangle \mathrm{Hx}$ was designed to contain a stabilised linker between HHIP-N and HHIP-C, comprising 7 point mutations (R185A, K186A, R189A, K204A, R210A, K211A and K213A) (see Supplementary Table 3 for used primers). This was performed to prevent interdomain proteolysis, as was observed previously for HHIP- $\Delta \mathrm{Hx}$ constructs ${ }^{26,27}$. HHIP constructs were expressed by transient transfection in HEK293T cells following a similar procedure as described in ${ }^{26}$. Briefly, HEK293T cells were grown in expanded-surface polystyrene roller bottles $\left(2125 \mathrm{~cm}^{2}\right.$, Greiner Bio-One). Per roller bottle, a transfection cocktail was prepared by incubating $1 \mathrm{~mL}$ of $1 \mathrm{mg} \mathrm{mL}^{-1} 25 \mathrm{kDa}$ branched polyethylenimine (Sigma Aldrich) with $0.5 \mathrm{mg}$ plasmid DNA in $50 \mathrm{~mL}$ serum-free DMEM for $10 \mathrm{~min}$ at room temperature. Prior to addition of the cocktail to the cells, the mixture was supplemented with $1 \mu \mathrm{g} \mathrm{mL}^{-1}$ final concentration of the glycosylation inhibitor kifunensine ${ }^{64}$. Proteins were then expressed for 3-5 days in DMEM supplemented with $2 \%$ (v/v) FBS and $2 \mathrm{mM}$ L-Glutamine/non-essential amino acids. Conditioned medium was dialysed against PBS and proteins were isolated via immobilised metal-affinity chromatography using a HisTrap HP ${ }^{\mathrm{TM}}$ column (GE Healthcare). All proteins were subsequently purified further via size exclusion chromatography in a buffer of $10 \mathrm{mM}$ HEPES pH 7.5, $150 \mathrm{mM} \mathrm{NaCl}$. In the case of HHIP-N and HHIP- $\triangle \mathrm{Hx}$, a further purification step utilising a HiTrap Heparin $\mathrm{HP}^{\mathrm{TM}}$ (GE Healthcare) column was added to remove co-eluting degradation products.

Heparin affinity chromatography. Purified HHIP constructs were loaded onto a $1 \mathrm{~mL}$ Heparin column (HiTrap Heparin HP: Life Technologies) equilibrated in $20 \mathrm{mM} \mathrm{NaCl}, 10 \mathrm{mM}$ HEPES pH $7.5(\sim 3 \mathrm{mS} / \mathrm{cm})$. Constructs were eluted over 20 column volumes using a linear gradient of elution buffer up to $1000 \mathrm{mM} \mathrm{NaCl}$, $10 \mathrm{mM}$ HEPES pH $7.5(\sim 85 \mathrm{mS} / \mathrm{cm})$. The elution of HHIP constructs was followed via absorption at $280 \mathrm{~nm}$. HHIP-N elutes at $520 \mathrm{mM} \mathrm{NaCl}, 10 \mathrm{mM}$ HEPES pH 7.5 $(\sim 44 \mathrm{mS} / \mathrm{cm}), \mathrm{HHIP}-\mathrm{C}$ at $480 \mathrm{mM} \mathrm{NaCl}, 10 \mathrm{mM}$ HEPES $\mathrm{pH} 7.5(\sim 41 \mathrm{mS} / \mathrm{cm})$ and HHIP- $\triangle \mathrm{Hx}$ at $590 \mathrm{mM} \mathrm{NaCl}, 10 \mathrm{mM}$ HEPES pH $7.5(\sim 50 \mathrm{mS} / \mathrm{cm})$.

Crystallisation and data collection. Prior to crystallisation trials, proteins were concentrated via ultrafiltration (HHIP-N, $6.8 \mathrm{mg} \mathrm{mL}^{-1}$; HHIP-C, $7.0 \mathrm{mg} \mathrm{mL}^{-1}$ ) and deglycosylated by addition of catalytic quantities of endoglycosidase $\mathrm{F} 1^{64}$ at a ratio of 1:250 (w/w) (HHIP:EndoF1). For HHIP co-crystallisation with GAGs, either $10 \mathrm{mM}$ SOS (Toronto Research Chemicals) or $1.5 \mathrm{mM} \mathrm{30-mer} \mathrm{heparin}$ (Iduron) was added to the concentrated protein. Nanolitre-scale crystallisation trials were performed using a Cartesian Technologies robot $(100 \mathrm{~nL}$ protein plus $100 \mathrm{~nL}$ reservoir solution) in 96-well Greiner plates ${ }^{65}$. The HHIP-N:SOS complex was crystallised in $0.1 \mathrm{M}$ imidazole/MES pH $6.5,0.1 \mathrm{M}$ carboxylic acids $(0.02 \mathrm{M}$ sodium formate, $0.02 \mathrm{M}$ ammonium acetate, $0.02 \mathrm{M}$ sodium citrate, $0.02 \mathrm{M}$ sodium oxamate and $0.02 \mathrm{M}$ potassium sodium tartrate), 10\% w/v PEG 20000 and $20 \% \mathrm{v} / \mathrm{v}$ PEG MME 550; apo HHIP-N was crystallised in 0.1 M HEPES pH 7.0, 0.2 M ammonium chloride, $0.02 \mathrm{M}$ hexamine cobalt (III) chloride, $0.002 \mathrm{M}$ SOS and $20 \%$ w/v PEG 6000. The HHIP-C:heparin complex was crystallised in $0.1 \mathrm{M}$ bis-Tris $\mathrm{pH}$ 6.5, $0.2 \mathrm{M}$ sodium citrate and 20\% w/v PEG 3350; the HHIP-C:SOS complex was crystallised in $0.1 \mathrm{M}$ Tris/Bicine $\mathrm{pH} 8.5,0.03 \mathrm{M}$ magnesium chloride, $0.03 \mathrm{M}$ calcium chloride, 10\% w/v PEG 20000 and 20\% v/v PEG MME 550. All crystals of HHIP were grown at $20{ }^{\circ} \mathrm{C}$.
Diffraction data were collected at a temperature of $100 \mathrm{~K}$ with crystals mounted within a liquid $\mathrm{N}_{2}$ cryo-stream. Crystals were treated with an appropriate cryoprotectant supplemented with reservoir solution (HHIP-N:SOS, 15\% glycerol; apo HHIP-N, 30\% ethylene glycol; HHIP-C:heparin, 30\% ethylene glycol; HHIPC:SOS, $20 \%$ glycerol) and flash-cooled in liquid $\mathrm{N}_{2}$ prior to data collection. Data were collected at Diamond Light Source, UK on beamlines I03 (HHIP-C:heparin, $\lambda=0.97631 \AA$ ), I04 (HHIP-N:SOS; HHIP-C:SOS, $\lambda=0.97949 \AA$ ) and I24 (apo HHIP-N, $\lambda=0.96862 \AA$ ). Data collection of HHIP-N for experimental phasing by the Sulphur-Single Anomalous Dispersion (S-SAD) method was carried out at $\lambda=1.7712 \AA$. This wavelength was selected to maximise the observed anomalous signal without compromising the transmission of X-rays ${ }^{66}$. To counteract the low anomalous signal from $S$ atoms at this wavelength, S-SAD data collection of HHIP$\mathrm{N}$ :SOS crystals was performed using inverse beam (I03) and mini-kappa goniometry-based (I04) approaches ${ }^{38,39}$. A total of 24 datasets on 8 different crystals were collected. In all other cases, data were collected using the standard rotation method. Diffraction data were scaled and merged using XIA2 ${ }^{67-72}$. The HHIP-C:heparin complex data was processed using the program DIALS in combination with $X I A 2^{73}$. In all cases, an inner shell with a $\mathrm{CC}_{1 / 2}$ of 0.30 was utilised in the selection of a high-resolution cut-off ${ }^{74}$.

Structure solution. We determined the structure of the HHIP-N:SOS complex by $\mathrm{S}-\mathrm{SAD}$ phasing. The sulphur substructure was determined using the HKL2MAP interface for the SHELX suite ${ }^{75,76}$. Using SHELXC, an anomalous signal to $4 \AA$ resolution was observed, and searching for 6 sulphur sites as disulphides in SHELXD gave a solution with $\mathrm{CC}_{\text {weak }}$ and $\mathrm{CC}_{\text {all }}$ statistics of 23.8 and 42.4 respectively. These SHELXD substructure coordinates were used as input files for an initial round of phasing in Phenix Autosol and Autobuild pipelines ${ }^{77,78}$. This enabled determination of a partial HHIP-N structure, with 87 of 182 residues built (Supplementary Fig. 1a). This partial model was then used as input for Molecular Replacement-SAD in Phaser $^{79}$. This allowed extension of the model to 98 residues placed in the asymmetric unit (Supplementary Fig. 1b). Manual building in $\operatorname{Coot}^{80}$ and initial refinement in Refmac5 $5^{81}$ enabled the tracing of 110 residues, also resulting in visible electron density for a SOS molecule (Supplementary Fig. 1c). The HHIP-N SOS complex was refined using iterative cycles of refinement in BUSTER 2.10.3 $3^{82}$ and Phenix ${ }^{83}$ (Supplementary Fig. 1d). Calculation of an anomalous difference map for the final HHIP-N:SOS structure identified 6 peaks at $4 \sigma$, corresponding to the 6 disulphide bonds (Supplementary Fig. 1e). The apo HHIP-N structure was solved by molecular replacement using the HHIP-N:SOS complex as a search model in Phaser $^{84}$ and subsequently refined using Phenix ${ }^{83}$.

Both the HHIP-C:heparin and HHIP-C:SOS complexes were solved by molecular replacement using the HHIP-C apo-structure (PDB ID: 2WFT; residues 213-670) $)^{26}$ as a search model in Phaser ${ }^{75}$. The HHIP-C:heparin complex was refined using Buster $2.10 .3^{82}$, and HHIP-C:SOS with Phenix ${ }^{83}$. For the HHIPC:heparin complex, 8 sugars were accounted for by the electron density per asymmetric unit. Restraints were generated for both pyranose constituents (IDS, SGN) of heparin using the program Privateer $^{85}$. For the HHIP-C:SOS complex, 2 SOS molecules were visible in the electron density per HHIP-C chain (2 HHIP-C chains per asymmetric unit).

Structure analysis. Stereochemical properties were assessed using the MolProbity server $^{86}$. Surface electrostatic potentials were generated using $A P B S^{87}$. Superpositions were calculated using PYMOL (www.pymol.org), which was also used to generate ray-traced images of protein structures for figures. Residues involved in interactions were identified using both the PDBSUM and PISA servers ${ }^{88,89}$. The solvent accessible radius was set to $1.4 \AA$ for the representation of all protein surfaces. Structural evolutionary analysis of CRDs was performed using $S H P^{90,91}$ and $P H Y L I P^{92}$ to assemble a phylogenetic tree (Supplementary Table 2). Structurebased sequence alignments of HHIP-N with evolutionarily-related CRDs were generated using UCSF Chimera ${ }^{93}$ and were prepared for publication using the program Aline ${ }^{94}$. Carbohydrate stereochemistry was validated with Privateer ${ }^{85}$.

Analytical ultracentrifugation (AUC). For AUC experiments HHIP-N and HHIP-C were dialysed into $10 \mathrm{mM}$ HEPES pH 7.5, $120 \mathrm{mM} \mathrm{NaCl}$. Experiments were performed at $20^{\circ} \mathrm{C}$ using a Beckman Optima XL-I analytical ultracentrifuge (Beckman Instruments) with absorbance optics at $280 \mathrm{~nm}$ and interference optics. HHIP-N samples were spun at a concentration of $0.7 \mathrm{mg} \mathrm{mL}^{-1}$, alone and with the addition of $0.09 \mathrm{mM} \mathrm{30-mer} \mathrm{heparin} \mathrm{(dp30,} \mathrm{Iduron).} \mathrm{HHIP-C} \mathrm{samples} \mathrm{were} \mathrm{spun}$ at a concentration of $1 \mathrm{mg} \mathrm{mL}^{-1}$, alone and with the addition of $0.16 \mathrm{mM} 30$-mer heparin (dp30, Iduron). As a control, heparin was spun alone in a separate chamber at the concentration specified and analysed using interference optics. Samples were contained within $12 \mathrm{~mm}$ Epon sector-shaped two-channel centerpieces and spun at $128,794 \times g$ (An60Ti rotor, Beckman Coulter Inc., CA), with 80 sample distribution scans taken in 6 min intervals, alongside interference optics. Absorbance data for scans 5-50 were analysed using the program SedFit for sizeand-shape distributions $c(s)$ and $(c(s, f r)$, where $f r$ is the frictional ratio and for a sphere $f r=1$ and for other species $f r>1)^{95}$. This enables the plotting of contour plots of $c(s, M)$, where $M$ is the weight of the protein. Due to the complex distributions observed in the case of HHIP-C:heparin, we present only $\mathrm{c}(s)$ distributions for HHIP-C experiments. For full clarity of solution molecular weights, 
$c(s, f r)$ distributions were calculated for HHIP-N. In all cases, a partial specific volume value of $0.73 \mathrm{~mL} \mathrm{~g}^{-1}$ was used.

Surface plasmon resonance. SPR experiments were performed using a Biacore T200 machine (GE Healthcare) in $10 \mathrm{mM}$ HEPES pH 7.5, $120 \mathrm{mM} \mathrm{NaCl}, 0.05 \%$ (v/ v) polysorbate 20 , at $25^{\circ} \mathrm{C}$. Proteins were buffer exchanged into running buffer and concentrations were calculated from the absorbance at $280 \mathrm{~nm}$ using predicted molar extinction coefficient values. Heparin (Iduron; average molecular weight $>9000 \mathrm{Da}$ ), heparan sulphate (HS) from porcine mucosa (Iduron) and chondroitin sulphate (CS) sodium salt from shark cartilage (Sigma) were biotinylated using EZlink Biotin-LC-Hydrazide (Thermo Fisher Scientific) in a solution containing 17\% (v/v) DMSO for 26 hours at $20^{\circ} \mathrm{C}$. GAGs were then extensively dialysed, first against water and then SPR running buffer $(120 \mathrm{mM} \mathrm{NaCl}, 10 \mathrm{mM}$ HEPES $\mathrm{pH} 7.5$, $0.05 \% \mathrm{v} / \mathrm{v}$ Tween 20), similar to a procedure described previously ${ }^{96}$. Biotinylated sugars were immobilised on CM5 sensor chips to which $3000 \mathrm{RU}$ of streptavidin were covalently coupled ${ }^{97}$. After each binding experiment, the chip was regenerated with running buffer supplemented with $1.5 \mathrm{M} \mathrm{NaCl}$ at $30 \mu \mathrm{Lmin}^{-1}$ for $120 \mathrm{~s}$. In all experiments, the trace returned to baseline following regeneration. HHIP constructs were injected at a flow rate of $5 \mu \mathrm{L} \mathrm{min}^{-1}$. All data were analysed using SCRUBBER2 (Biologic) and GraphPad Prism Version 6.04 (GraphPad Software, La Jolla California USA). Best-fit binding curves were calculated using non-linear curve fitting of a one-site-total binding model $\left(Y=\left[R_{\max }{ }^{*} X /\left(K_{D}+X\right)\right]+\right.$ $N S^{*} X+$ Background], where $\mathrm{X}$ is analyte concentration and the level of nonspecific binding is assumed to be proportional to the analyte concentration; hence NS equals the slope of non-specific binding). The background value was set to zero as the data had been previously referenced. $R_{\max }$ and $K_{D}$ values quoted are determined for the specific binding portion only.

Isothermal titration calorimetry. Experiments were performed using a MicroCal PEAQ-ITC (Malvern) at $25^{\circ} \mathrm{C}$ in $10 \mathrm{mM}$ HEPES, $\mathrm{pH} 7.5,150 \mathrm{mM} \mathrm{NaCl}$ and $3 \%$ isopropanol, with a differential power of $10 \mu \mathrm{cal} \mathrm{s}{ }^{-1}$ and stirring at $750 \mathrm{rpm}$. Experiments consisted of an initial test injection of $0.4 \mu \mathrm{L}$, followed 150 s later by 18 injections of $2 \mu \mathrm{L}$, spaced $150 \mathrm{~s}$ apart. Owing to the low solubility of cholesterol, a PEG-cholesterol was used for affinity measurements. HHIPN was dialysed against $0.15 \mathrm{M} \mathrm{NaCl}, 10 \mathrm{mM}$ HEPES and 3\% isopropanol, final $\mathrm{pH}$ 7.5. Lyophilized PEG-cholesterol and PEG200 were resuspended in dialysis solution to a concentration of $1 \mathrm{mM}$. Protein concentrations were determined from the absorbance at $280 \mathrm{~nm}$ using calculated molar extinction coefficients. Cell concentrations of $9 \mu \mathrm{M}$ HHIPN protein and syringe concentrations of $1 \mathrm{mM}$ PEG-cholesterol or PEG200 were used for all experiments. Thermograms were integrated and corrected for heats of dilution using PEAQ-ITC analysis software (Malvern). Isotherms were fitted with the $A+B \rightleftharpoons A B$ model, where cell and syringe concentrations and baselines of each experiment were fitted locally. All figures were prepared using PEAQ-ITC analysis software (Malvern).

Immunofluorescence microscopy. HEK 293 T cells were seeded at a concentration of 10,000 cells $\mathrm{mL}^{-1}$ in poly-D-lysine coated $35 \mathrm{~mm}$ dishes (MatTek) and incubated at $37^{\circ} \mathrm{C}, 5 \% \mathrm{CO}_{2}$ for $18 \mathrm{~h}$. Media was exchanged to reduce serum concentration from 10 to $2 \%$ and cells were transfected with $2 \mu \mathrm{g}$ DNA of HAtagged constructs using lipofectamine at a ratio of 1:2. 6 hours following transfection, cellular growth medium was further lowered to $0.5 \%$ serum and cells were further incubated for 2 days. Media was removed and cells were washed with PBS before fixation for 15 min with $3 \%$ para-formaldehyde and quenching in $0.3 \mathrm{M}$ glycine for $3 \mathrm{~min}$. Fixed cells were stored overnight at $4{ }^{\circ} \mathrm{C}$ in PBS. For the staining process, fixed cells were blocked for 10 min using $1 \% \mathrm{BSA} /$ PBS, before HA tag probing using a Mouse HA Epitope Tag Antibody (Thermo Fisher) at $1 \mu \mathrm{g} \mathrm{mL}^{-1}$ in $1 \% \mathrm{BSA} / \mathrm{PBS}$ at $25^{\circ} \mathrm{C}$ for $1 \mathrm{~h}$. Cells were washed in PBS 3 times for $10 \mathrm{~min}$, before incubation with an Alexa Fluor ${ }^{\circledR}$ 633-conjugated Goat anti-Mouse IgG antibody $\left(\lambda_{\text {ex }}: 633 \mathrm{~nm} ; \lambda_{\text {em }}: 647 \mathrm{~nm}\right.$ ) (Thermo Fisher) at a concentration of $2 \mu \mathrm{g} \mathrm{mL}^{-1}$ in $1 \% \mathrm{BSA} / \mathrm{PBS}$ for $1 \mathrm{~h}$ at $25^{\circ} \mathrm{C}$. Excess antibody was removed via washing 3 times in PBS for $10 \mathrm{~min}$, with one final wash containing $0.5 \mu \mathrm{g} \mathrm{mL}^{-1}$ Hoechst 33342 nuclear stain $\left(\lambda_{\text {ex }}: 353 \mathrm{~nm} ; \lambda_{\text {em }}: 483 \mathrm{~nm}\right)^{98}$. Immunofluorescence was detected using a Leica TCS SP8 WLL Confocal SMD Microscope. Images were processed in Fiji ${ }^{99}$.

Multiangle light scattering (MALS). A total of $100 \mu \mathrm{L}$ protein samples were injected onto an S200 10/30 column (GE Healthcare) equilibrated in a running buffer of $10 \mathrm{mM}$ HEPES pH 7.5, $150 \mathrm{mM} \mathrm{NaCl}$ over a concentration range of 48-192 $\mu \mathrm{M}$. A Wyatt Dawn HELEOS-II MALS detector and Wyatt Optilab rEX refractive index monitor recorded both the refractive index and light scattering once separated via SEC. ASTRA software (Wyatt Technology) was used for data analysis.

HHIP-C mutant heparin affinity chromatography. HHIP-C constructs $(200 \mu \mathrm{g} /$ run) were loaded onto a $1 \mathrm{~mL}$ heparin column (HiTrap heparin HP, GE Healthcare) in a buffer of $10 \mathrm{mM}$ HEPES pH 7.5, $40 \mathrm{mM} \mathrm{NaCl}(\sim 5 \mathrm{mS} / \mathrm{cm})$ and eluted over 30 column volumes with a gradient of elution buffer up to $10 \mathrm{mM}$ HEPES $\mathrm{pH}$ $7.5,1000 \mathrm{mM} \mathrm{NaCl}(\sim 87 \mathrm{mS} / \mathrm{cm})$. Elution was followed by absorption at $280 \mathrm{~nm}$. The HHIP-C Glu mutant eluted from the column at $\sim 240 \mathrm{mM} \mathrm{NaCl}, 10 \mathrm{mM}$
HEPES pH $7.5(22 \mathrm{mS} / \mathrm{cm})$. The dual GAG site mutant $(\triangle \mathrm{GAG}$ sites $1+2)$ eluted at $\sim 320 \mathrm{mM} \mathrm{NaCl}, 10 \mathrm{mM}$ HEPES pH $7.5(29 \mathrm{mS} / \mathrm{cm})$. The $\beta$-propeller interface GAG mutant ( $\triangle \mathrm{GAG}$ site 1 ) eluted at $\sim 400 \mathrm{mM} \mathrm{NaCl}, 10 \mathrm{mM}$ HEPES pH 7.5 (36 $\mathrm{mS} / \mathrm{cm}$ ) and the EGF interface GAG mutant $(\Delta \mathrm{GAG}$ site 2) eluted at $\sim 410 \mathrm{mM}$ $\mathrm{NaCl}, 10 \mathrm{mM}$ HEPES pH $7.5(37 \mathrm{mS} / \mathrm{cm})$. Wild-type HHIP-C eluted at $\sim 450 \mathrm{mM}$ $\mathrm{NaCl}, 10 \mathrm{mM}$ HEPES pH $7.5(40 \mathrm{mS} / \mathrm{cm})$.

Generation of NIH/3T3 Ext/3 ${ }^{-/}$fibroblasts. Clonal EXTL3 knockout cell lines were generated using a double-cut CRISPR strategy. Guides were cloned into pSpCas9(BB)-2A-GFP (PX458; Addgene \#48138) and pSpCas9(BB)-2A-mCherry Plasmids were co-transfected into NIH/3T3 Flp-Ins using X-tremeGENE 9 (Roche). Four days post-transfection, GFP and mCherry double-positive single cells were sorted into 96-well plates using a Sony Cell Sorter Model SH800S. To screen clonal cell lines, we used PCR to detect successful excision of the genomic DNA between the two sgRNA cut sites (Supplementary Fig. 12, Supplementary Table 3).

HS20 cell staining of CRISPR generated EXTL3 knockouts. To confirm that Extl3 ${ }^{-1-}$ NIH/3T3 cells had reduced cell surface Heparan Sulfate Proteoglycans (HSPGs), we stained intact cells with a previously characterized HS20 scFv antibody (fused to a 1D4 epitope tag), which recognizes a Heparan Sulphate chains attached to HSPG ${ }^{54,55}$. Cells were trypsinized and resuspended in Staining Buffer (SB: PBS $+2 \%$ BSA $+0.05 \%$ sodium azide) at a density of 500,000 cells $/ 100 \mu \mathrm{L}$ buffer. Cells were then spun down and resuspended in SB with 5\% donkey serum. Following a ten-minute room temperature incubation, cells were spun down, resuspended in $50 \mu \mathrm{L} \mathrm{SB}$ containing HS20-1D4 tag (1:20.83 dilution), and incubated for 30 minutes at $4{ }^{\circ} \mathrm{C}$. Samples were then washed twice with SB, before being incubated for $30 \mathrm{~min}$ at $4{ }^{\circ} \mathrm{C}$ in anti-1D4 monoclonal antibody diluted in SB (1:500; The University of British Columbia). After incubation with anti-1D4 antibody, cells were again washed twice with SB. Samples were then incubated in donkey antimouse antibody coupled to Alexa Fluor 594 diluted in SB (1:500; ThermoFisher Scientific A-21203) for 30 minutes at $4{ }^{\circ} \mathrm{C}$. Cells were then washed three times with SB before being resuspended in PBS for flow cytometry analysis. Flow cytometry was performed on a BD Acuri C6 Flow Cytometer using a $552 \mathrm{~nm}$ laser for excitation and a 610/20 nm bandpass filter to collect emitted light. Forward and side scatter plots were used to select a population of live, mostly single cells, which were then analyzed. A population of at least 7500 selected cells was analyzed for each sample.

HH signalling assay. Gli1 mRNA measurements were used as a readout for $\mathrm{HH}$ pathway activation and were performed as in ${ }^{7}$. Briefly, NIH/3T3 cells (ATCC, CRL-1658) were grown to confluency and then serum-starved for $24 \mathrm{~h}$ via reduction of serum to $0.5 \%$ NBCS. The HH pathway was either induced by adding $50 \mathrm{nM}$ purified $\mathrm{ShhN}_{\mathrm{C} 24 \mathrm{II}} 12,100$, or NIH/3T3 cells were co-cultured with the fulllength HH-producing human cell line PANC-1 (ATCC, CRL-1469) ${ }^{48}$, with addition of HHIP-C constructs or HHIP- $\Delta \mathrm{Hx}$ at $100 \mathrm{nM}$ in each case. For HH pathway stimulation by the palmitoylated N-terminal ShhN peptide (Palm-ShhN15), peptides were added at $10 \mu \mathrm{M}$ and HHIP- $\Delta \mathrm{Hx}$ at $1 \mu \mathrm{M}(n=2)$. Reverse transcription quantitative PCR (RT-qPCR) was carried out with the Power SYBR ${ }^{\circledast}$ Green Cellsto-CT ${ }^{\mathrm{TM}}$ kit (Life Technologies) according to manufacturer's instructions. Every experiment was performed with two biological replicates and three technical replicates and results were calculated according to the $\Delta \Delta C T$ method and maximal pathway activation was normalised to 100. The PCR primers for Gli1 (forward primer, $5^{\prime}$-ccaagccaactttatgtcaggg- $3^{\prime}$; reverse primer, $5^{\prime}$-agcccgcttctttgttaatttga- $3^{\prime}$ ); and Gapdh (forward primer, $5^{\prime}$-agtggcaaagtggagatt- $3^{\prime}$; reverse primer, $5^{\prime}$-gtggagtcatactggaaca- $\left.3^{\prime}\right)$ are specific for murine DNAs.

Reporting summary. Further information on research design is available in the Nature Research Reporting Summary linked to this article.

\section{Data availability}

Atomic coordinates and structure factors for HHIP-N:SOS, HHIP-N apo, HHIPC:heparin and HHIP-C:SOS have been deposited in the Protein Data Bank (PDB) (www.rcsb.org) under accession numbers 7PGK, 7PGL, 7PGM and 7PGN, respectively. Any other data supporting the findings of this manuscript are available from the corresponding author upon reasonable request. Source data are provided with this paper.

Received: 29 April 2020; Accepted: 19 November 2021; Published online: 09 December 2021

\section{References}

1. Briscoe, J. \& Thérond, P. P. The mechanisms of Hedgehog signalling and its roles in development and disease. Nat. Rev. Mol. Cell Biol. 14, 418-431 (2013). 
2. Kong, J. H., Siebold, C. \& Rohatgi, R. Biochemical mechanisms of vertebrate hedgehog signaling. Development (Cambridge, England) 146, https://doi.org/ 10.1242/dev.166892 (2019).

3. Rubin, L. L. \& de Sauvage, F. J. Targeting the Hedgehog pathway in cancer. Nat. Rev. Drug Disco. 5, 1026-1033 (2006).

4. Wu, F., Zhang, Y., Sun, B., McMahon, A. P. \& Wang, Y. Hedgehog signaling: from basic biology to cancer therapy. Cell Chem. Biol. 24, 252-280 (2017).

5. Porter, J. A. et al. Hedgehog patterning activity: role of a lipophilic modification mediated by the carboxy-terminal autoprocessing domain. Cell 86, 21-34 (1996)

6. Pepinsky, R. B. et al. Identification of a palmitic acid-modified form of human Sonic hedgehog. J. Biol. Chem. 273, 14037-14045 (1998).

7. Gong, X. et al. Structural basis for the recognition of Sonic Hedgehog by human Patched1. Science (New York, N.Y.) 361, https://doi.org/10.1126/ science.aas8935 (2018).

8. Qi, X., Schmiege, P., Coutavas, E., Wang, J. \& Li, X. Structures of human Patched and its complex with native palmitoylated sonic hedgehog. Nature 560, 128-132 (2018).

9. Qi, X., Schmiege, P., Coutavas, E. \& Li, X. Two Patched molecules engage distinct sites on Hedgehog yielding a signaling-competent complex. Science (New York, N.Y.) 362, https://doi.org/10.1126/science.aas8843 (2018).

10. Zhang, Y. et al. Structural basis for cholesterol transport-like activity of the Hedgehog receptor patched. Cell 175, 1352-1364.e1314 (2018).

11. Qi, C., Di Minin, G., Vercellino, I., Wutz, A. \& Korkhov, V. M. Structural basis of sterol recognition by human hedgehog receptor PTCH1. Sci. Adv. 5, eaaw6490 (2019)

12. Rudolf, A. F. et al. The morphogen Sonic hedgehog inhibits its receptor Patched by a pincer grasp mechanism. Nat. Chem. Biol. 15, 975-982 (2019).

13. Luchetti, G. et al. Cholesterol activates the G-protein coupled receptor Smoothened to promote Hedgehog signaling. eLife 5, https://doi.org/10.7554/ eLife.20304 (2016).

14. Huang, P. et al. Cellular cholesterol directly activates smoothened in Hedgehog signaling. Cell 166, 1176-1187.e1114 (2016).

15. Myers, B. R. et al. Hedgehog pathway modulation by multiple lipid binding sites on the smoothened effector of signal response. Dev. cell 26, 346-357 (2013).

16. Bidet, M. et al. The hedgehog receptor patched is involved in cholesterol transport. PLoS ONE 6, e23834 (2011).

17. Beachy, P. A., Hymowitz, S. G., Lazarus, R. A., Leahy, D. J. \& Siebold, C. Interactions between Hedgehog proteins and their binding partners come into view. Genes Dev. 24, 2001-2012 (2010)

18. Rubin, J. B., Choi, Y. \& Segal, R. A. Cerebellar proteoglycans regulate sonic hedgehog responses during development. Dev. (Camb., Engl.) 129, 2223-2232 (2002).

19. Whalen, D. M., Malinauskas, T., Gilbert, R. J. C. \& Siebold, C. Structural insights into proteoglycan-shaped Hedgehog signaling. Proc. Natl Acad. Sci. USA 110, 16420-16425 (2013).

20. Eaton, S. Multiple roles for lipids in the Hedgehog signalling pathway. Nat. Rev. Mol. Cell Biol. 9, 437-445 (2008).

21. Ohlig, S. et al. Sonic hedgehog shedding results in functional activation of the solubilized protein. Dev. Cell 20, 764-774 (2011).

22. McLellan, J. S. et al. The mode of Hedgehog binding to Ihog homologues is not conserved across different phyla. Nature 455, 979-983 (2008).

23. Kavran, J. M., Ward, M. D., Oladosu, O. O., Mulepati, S. \& Leahy, D. J. All mammalian Hedgehog proteins interact with cell adhesion molecule, downregulated by oncogenes (CDO) and brother of $\mathrm{CDO}$ (BOC) in a conserved manner. J. Biol. Chem. 285, 24584-24590 (2010).

24. Allen, B. L. et al. Overlapping roles and collective requirement for the coreceptors GAS1, CDO, and BOC in SHH pathway function. Dev. Cell $\mathbf{2 0}$, 775-787 (2011).

25. Izzi, L. et al. Boc and Gas1 each form distinct Shh receptor complexes with Ptch1 and are required for Shh-mediated cell proliferation. Developmental cell 20, 788-801 (2011).

26. Bishop, B. et al. Structural insights into hedgehog ligand sequestration by the human hedgehog-interacting protein HHIP. Nat. Struct. Mol. Biol. 16, 698-703 (2009).

27. Bosanac, I. et al. The structure of SHH in complex with HHIP reveals a recognition role for the Shh pseudo active site in signaling. Nat. Struct. Mol. Biol. 16, 691-697 (2009).

28. Holtz, A. M. et al. Secreted HHIP1 interacts with heparan sulfate and regulates Hedgehog ligand localization and function. J. Cell Biol. 209, 739-758 (2015).

29. Kwong, L., Bijlsma, M. F. \& Roelink, H. Shh-mediated degradation of Hhip allows cell autonomous and non-cell autonomous Shh signalling. Nat. Commun. 5, 4849 (2014)

30. Holtz, A. M. et al. Essential role for ligand-dependent feedback antagonism of vertebrate hedgehog signaling by PTCH1, PTCH2 and HHIP1 during neural patterning. Dev. (Camb., Engl.) 140, 3423-3434 (2013).
31. Chuang, P. T., Kawcak, T. \& McMahon, A. P. Feedback control of mammalian Hedgehog signaling by the Hedgehog-binding protein, Hip1, modulates Fgf signaling during branching morphogenesis of the lung. Genes Dev. 17, 342-347 (2003).

32. Chuang, P. T. \& McMahon, A. P. Vertebrate Hedgehog signalling modulated by induction of a Hedgehog-binding protein. Nature 397, 617-621 (1999).

33. Wilson, N. H. \& Stoeckli, E. T. Sonic hedgehog regulates its own receptor on postcrossing commissural axons in a glypican1-dependent manner. Neuron 79, 478-491 (2013)

34. Olsen, C. L., Hsu, P. P., Glienke, J., Rubanyi, G. M. \& Brooks, A. R. Hedgehoginteracting protein is highly expressed in endothelial cells but down-regulated during angiogenesis and in several human tumors. BMC Cancer 4, 43 (2004).

35. Prokopenko, D. et al. Whole-genome sequencing in severe chronic obstructive pulmonary disease. Am. J. Respir. Cell Mol. Biol. 59, 614-622 (2018).

36. Bazan, J. F. \& de Sauvage, F. J. Structural ties between cholesterol transport and morphogen signaling. Cell 138, 1055-1056 (2009).

37. Aricescu, A. R., Lu, W. \& Jones, E. Y. A time- and cost-efficient system for high-level protein production in mammalian cells. Acta Crystallogr. Sect. D., Biol. Crystallogr. 62, 1243-1250 (2006).

38. El Omari, K. et al. Pushing the limits of sulfur SAD phasing: de novo structure solution of the N-terminal domain of the ectodomain of HCV E1. Acta Crystallogr. Sect. D., Biol. Crystallogr. 70, 2197-2203 (2014).

39. Hendrickson, W. A. Anomalous diffraction in crystallographic phase evaluation. Q. Rev. biophysics 47, 49-93 (2014).

40. Debreczeni, J. E., Bunkoczi, G., Ma, Q., Blaser, H. \& Sheldrick, G. M. In-house measurement of the sulfur anomalous signal and its use for phasing. Acta Crystallogr. Sect. D. 59, 688-696 (2003).

41. Chen, C. et al. Structural basis for molecular recognition of folic acid by folate receptors. Nature 500, 486-489 (2013).

42. Monaco, H. L. Crystal structure of chicken riboflavin-binding protein. $E M B O$ J. 16, 1475-1483 (1997).

43. Byrne, E. F. X. et al. Structural basis of Smoothened regulation by its extracellular domains. Nature, https://doi.org/10.1038/nature18934 (2016).

44. Kwon, H. J. et al. Structure of N-terminal domain of NPC1 reveals distinct subdomains for binding and transfer of cholesterol. Cell 137, 1213-1224 (2009).

45. Janda, C. Y., Waghray, D., Levin, A. M., Thomas, C. \& Garcia, K. C. Structural basis of Wnt recognition by Frizzled. Science (N. Y.) 337, 59-64 (2012).

46. Nachtergaele, S. et al. Structure and function of the Smoothened extracellular domain in vertebrate Hedgehog signaling. eLife 2, e01340 (2013).

47. Han, L. et al. Divergent evolution of vitamin B9 binding underlies Junomediated adhesion of mammalian gametes. Curr. Biol. 26, R100-R101 (2016).

48. Konitsiotis, A. D. et al. Attenuation of Hedgehog acyltransferase-catalyzed sonic Hedgehog palmitoylation causes reduced signaling, proliferation and invasiveness of human carcinoma cells. PLoS ONE 9, e89899 (2014).

49. Tukachinsky, H., Petrov, K., Watanabe, M. \& Salic, A. Mechanism of inhibition of the tumor suppressor Patched by Sonic Hedgehog. Proc. Natl Acad. Sci. USA, 201606719, https://doi.org/10.1073/pnas.1606719113 (2016).

50. Luque, I. \& Freire, E. Structure-based prediction of binding affinities and molecular design of peptide ligands. Methods Enzymol. 295, 100-127 (1998).

51. Mulloy, B. \& Forster, M. J. Conformation and dynamics of heparin and heparan sulfate. Glycobiology 10, 1147-1156 (2000).

52. Waksman, G. \& Herr, A. B. New insights into heparin-induced FGF oligomerization. Nat. Struct. Biol. 5, 527-530 (1998).

53. Lebensohn, A. M. \& Rohatgi, R. R-spondins can potentiate WNT signaling without LGRs. eLife 7, https://doi.org/10.7554/eLife.33126 (2018).

54. Dubey, R. et al. R-spondins engage heparan sulfate proteoglycans to potentiate WNT signaling. eLife 9, https://doi.org/10.7554/eLife.54469 (2020).

55. Gao, W. et al. Inactivation of Wnt signaling by a human antibody that recognizes the heparan sulfate chains of glypican-3 for liver cancer therapy. Hepatology 60, 576-587 (2014).

56. Janda, C. Y. \& Garcia, K. C. Wnt acylation and its functional implication in Wnt signalling regulation. Biochem Soc. Trans. 43, 211-216 (2015).

57. Kowatsch, C., Woolley, R. E., Kinnebrew, M., Rohatgi, R. \& Siebold, C. Structures of vertebrate Patched and Smoothened reveal intimate links between cholesterol and Hedgehog signalling. Curr. Opin. Struct. Biol. 57, 204-214 (2019).

58. Qi, X. \& Li, X. Mechanistic insights into the generation and transduction of Hedgehog signaling. Trends Biochem Sci. 45, 397-410 (2020).

59. Coulombe, J., Traiffort, E., Loulier, K., Faure, H. \& Ruat, M. Hedgehog interacting protein in the mature brain: membrane-associated and soluble forms. Mol. Cell. Neurosci. 25, 323-333 (2004).

60. Lin, X. Functions of heparan sulfate proteoglycans in cell signaling during development. Dev. (Camb., Engl.) 131, 6009-6021 (2004).

61. Maun, H. R. et al. Hedgehog pathway antagonist 5E1 binds hedgehog at the pseudo-active site. J. Biol. Chem. 285, 26570-26580 (2010).

62. Bailey, J. M. et al. Sonic hedgehog promotes desmoplasia in pancreatic cancer. Clin. Cancer Res 14, 5995-6004 (2008). 
63. Xu, D. \& Esko, J. D. Demystifying heparan sulfate-protein interactions. Annu Rev. Biochem 83, 129-157 (2014).

64. Chang, V. T. et al. Glycoprotein structural genomics: solving the glycosylation problem. Struct. (Lond., Engl.: 1993) 15, 267-273 (2007).

65. Walter, T. S. et al. A procedure for setting up high-throughput nanolitre crystallization experiments. Crystallization workflow for initial screening, automated storage, imaging and optimization. Acta Crystallogr. Sect. D. Biol. Crystallogr. 61, 651-657 (2005).

66. Liu, Z. J. et al. A multi-dataset data-collection strategy produces better diffraction data. Acta Crystallogr. Sect. A, Found. Crystallogr. 67, 544-549 (2011).

67. Evans, P. Scaling and assessment of data quality. Acta Crystallogr. Sect. D. Biol. Crystallogr. 62, 72-82 (2006).

68. Kabsch, W. Automatic indexing of rotation diffraction patterns. J. Appl. Crystallogr. 21, 67-72 (1988).

69. Leslie, A. G. The integration of macromolecular diffraction data. Acta Crystallogr. Sect. D., Biol. Crystallogr. 62, 48-57 (2006).

70. Sauter, N. K., Grosse-Kunstleve, R. W. \& Adams, P. D. Robust indexing for automatic data collection. J. Appl. Crystallogr. 37, 399-409 (2004).

71. Winter, G. xia2: an expert system for macromolecular crystallography data reduction. J. Appl. Crystallogr. 43, 186-190 (2010).

72. Zhang, Z., Sauter, N. K., van den Bedem, H., Snell, G. \& Deacon, A. M. Automated diffraction image analysis and spot searching for high-throughput crystal screening. J. Appl. Crystallogr. 39, 112-119 (2006).

73. Gildea, R. J. et al. New methods for indexing multi-lattice diffraction data. Acta Crystallogr. Sect. D. Biol. Crystallogr. 70, 2652-2666 (2014).

74. Karplus, P. A. \& Diederichs, K. Linking crystallographic model and data quality. Science (N. Y.) 336, 1030-1033 (2012).

75. Pape, T. \& Schneider, T. R. HKL2MAP: a graphical user interface for macromolecular phasing with SHELX programs. J. Appl. Crystallogr. 37, 843-844 (2004).

76. Sheldrick, G. M. Experimental phasing with SHELXC/D/E: combining chain tracing with density modification. Acta Crystallogr. Sect. D. Biol. Crystallogr. 66, 479-485 (2010).

77. Terwilliger, T. C. et al. Decision-making in structure solution using Bayesian estimates of map quality: the PHENIX AutoSol wizard. Acta Crystallogr. Sect. D. Biol. Crystallogr. 65, 582-601 (2009).

78. Terwilliger, T. C. et al. Iterative model building, structure refinement and density modification with the PHENIX AutoBuild wizard. Acta Crystallogr. Sect. D. Biol. Crystallogr. 64, 61-69 (2008).

79. Read, R. J. \& McCoy, A. J. Using SAD data in Phaser. Acta Crystallogr. Sect. D. Biol. Crystallogr. 67, 338-344 (2011)

80. Emsley, P. \& Cowtan, K. Coot: model-building tools for molecular graphics. Acta Crystallogr. Sect. D. Biol. Crystallogr. 60, 2126-2132 (2004).

81. Murshudov, G. N. et al. REFMAC5 for the refinement of macromolecular crystal structures. Acta Crystallogr. Sect. D. 67, 355-367 (2011)

82. Smart, O. S. et al. Exploiting structure similarity in refinement: automated NCS and target-structure restraints in BUSTER. Acta Crystallogr. Sect. D. Biol. Crystallogr. 68, 368-380 (2012)

83. Adams, P. D. et al. PHENIX: a comprehensive Python-based system for macromolecular structure solution. Acta Crystallogr. Sect. D. Biol. Crystallogr. 66, 213-221 (2010)

84. McCoy, A. J. et al. Phaser crystallographic software. J. Appl Crystallogr 40, 658-674 (2007).

85. Agirre, J. et al. Privateer: software for the conformational validation of carbohydrate structures. Nat. Struct. Mol. Biol. 22, 833-834 (2015).

86. Davis, I. W. et al. MolProbity: all-atom contacts and structure validation for proteins and nucleic acids. Nucleic acids Res. 35, W375-W383 (2007).

87. Baker, N. A., Sept, D., Joseph, S., Holst, M. J. \& McCammon, J. A Electrostatics of nanosystems: application to microtubules and the ribosome. Proc. Natl Acad. Sci. USA 98, 10037-10041 (2001).

88. Krissinel, E. \& Henrick, K. Inference of macromolecular assemblies from crystalline state. J. Mol. Biol. 372, 774-797 (2007).

89. Laskowski, R. A. PDBsum: summaries and analyses of PDB structures. Nucleic Acids Res. 29, 221-222 (2001).

90. Riffel, N. et al. Atomic resolution structure of moloney murine leukemia virus matrix protein and its relationship to other retroviral matrix proteins. Struct. (Lond., Engl.: 1993) 10, 1627-1636 (2002).

91. Stuart, D. I., Levine, M., Muirhead, H. \& Stammers, D. K. Crystal structure of cat muscle pyruvate kinase at a resolution of 2.6 A. J. Mol. Biol. 134, 109-142 (1979).

92. Felsenstein, J. PHYLIP: Phylogeny Inference Package. Version 3.2. Joel Felsenstein. 64, 539-541, (1989).

93. Pettersen, E. F. et al. UCSF Chimera-a visualization system for exploratory research and analysis. J. Comput. Chem. 25, 1605-1612 (2004).
94. Bond, C. S. \& Schuttelkopf, A. W. ALINE: a WYSIWYG protein-sequence alignment editor for publication-quality alignments. Acta Crystallogr. Sect. D., Biol. Crystallogr. 65, 510-512 (2009).

95. Brown, P. H. \& Schuck, P. Macromolecular size-and-shape distributions by sedimentation velocity analytical ultracentrifugation. Biophys. J. 90, 4651-4661 (2006).

96. Malinauskas, T., Aricescu, A. R., Lu, W., Siebold, C. \& Jones, E. Y. Modular mechanism of Wnt signaling inhibition by Wnt inhibitory factor 1. Nat. Struct. Mol. Biol. 18, 886-893 (2011).

97. O'Callaghan C, A. et al. BirA enzyme: production and application in the study of membrane receptor-ligand interactions by site-specific biotinylation. Anal. Biochem. 266, 9-15 (1999).

98. Chazotte, B. Labeling nuclear DNA with hoechst 33342. Cold Spring Harbor protocols 2011, pdb.prot5557 (2011).

99. Schindelin, J. et al. Fiji: an open-source platform for biological-image analysis. Nat. Methods 9, 676-682 (2012).

100. Taylor, F. R. et al. Enhanced potency of human sonic hedgehog by hydrophobic modification. Biochemistry 40, 4359-4371 (2001).

\section{Acknowledgements}

We thank the staff of beamlines I03, I04, and I24 at the Diamond Light Source, UK for assistance, and T. Walter, K. Harlos and D. Staunton for technical support. C.S. was supported by grants from Cancer Research UK (C20724/A14414 and C20724/A26752) and a European Research Council grant (647278). D.F.C. was supported by a grant from the National Institutes of Health (HL067773) and the Taylor Family Institute for Innovative Psychiatric Research. R.R. was supported by grants from the National Institutes of Health (GM118082 and GM106078). S.C.G. was supported by a Wellcome Trust PhD studentship (099675/Z/12/Z). The Wellcome Centre for Human Genetics, Oxford, is funded by Wellcome Trust Core Award 203852/Z/16/2.

\section{Author contributions}

C.S., S.C.G., and R.A.S. designed the project. S.C.G. and B.B. expressed and purified the proteins for crystallization and carried out biophysical experiments. T.M. contributed to protein production. S.C.G. and R.J.C.G. performed AUC analysis. S.C.G. crystallized the proteins. S.C.G. and C.S. collected and processed the X-ray data. S.C.G., K.E.O. and C.S solved and refined the crystal structures. R.A.S. conceived the cellular experiments and performed SHH signalling assays. E.J.I., R.D., and R.R. generated the extl-/- cell line, M.Q. and D.F.C. synthesized the PEG-cholesterol. S.C.G. and C.S. prepared the manuscript with input from all authors.

\section{Competing interests}

The authors declare no competing interests.

\section{Additional information}

Supplementary information The online version contains supplementary material available at https://doi.org/10.1038/s41467-021-27475-2.

Correspondence and requests for materials should be addressed to Christian Siebold.

Peer review information Nature Communications thanks the anonymous reviewer(s) for their contribution to the peer review of this work.

Reprints and permission information is available at http://www.nature.com/reprints

Publisher's note Springer Nature remains neutral with regard to jurisdictional claims in published maps and institutional affiliations.

Open Access This article is licensed under a Creative Commons Attribution 4.0 International License, which permits use, sharing, adaptation, distribution and reproduction in any medium or format, as long as you give appropriate credit to the original author(s) and the source, provide a link to the Creative Commons license, and indicate if changes were made. The images or other third party material in this article are included in the article's Creative Commons license, unless indicated otherwise in a credit line to the material. If material is not included in the article's Creative Commons license and your intended use is not permitted by statutory regulation or exceeds the permitted use, you will need to obtain permission directly from the copyright holder. To view a copy of this license, visit http://creativecommons.org/ licenses/by/4.0/

(C) The Author(s) 2021 